\title{
Sizing extracellular vesicles using membrane dyes and a single molecule-sensitive flow analyzer
}

\author{
Luca A. Andronico, ${ }^{\dagger}$ Yifei Jiang, ${ }^{\dagger}$ Seung-Ryoung Jung, ${ }^{\dagger}$ Bryant S. Fujimoto, ${ }^{\dagger}$ Lucia Vojtech, ${ }^{\ddagger}$ and Daniel T. Chiu ${ }^{* \dagger}$ \\ †Department of Chemistry, University of Washington, Seattle, Washington 98195, USA. \\ ‡Department of Obstetrics and Gynecology, University of Washington, Seattle, USA. \\ *Correspondence and requests for materials should be addressed to D.T.C. (email: chiu@chem.washington.edu).
}

\section{Table of Contents}

1) Molecular structure and bulk emission of membrane dyes;

pp. 2-3

2) Supporting figures for dye aggregation in buffer;

pp. 4

3) Procedure and supporting figures for optimization of vesicles staining;

pp. $5-7$

4) Schematic of pseudo code used for flow data analysis;

pp. 8

5) Calculation of total uncertainty on size estimate;

pp. 9-14

6) Supporting figures for the selection of membrane dye;

pp. 15

7) Proposed mechanism of vesicles staining with Di-8-ANEPPS;

pp. 16-17

8) Bulk emission of Di-8-ANEPPS and Cryo-EM images;

pp. 18

9) Parameters of size distributions obtained during the staining optimization;

pp. 19

10) Supporting figures for the screening of optimal conditions for SE staining;

pp. $20-25$

11) References

pp.26 

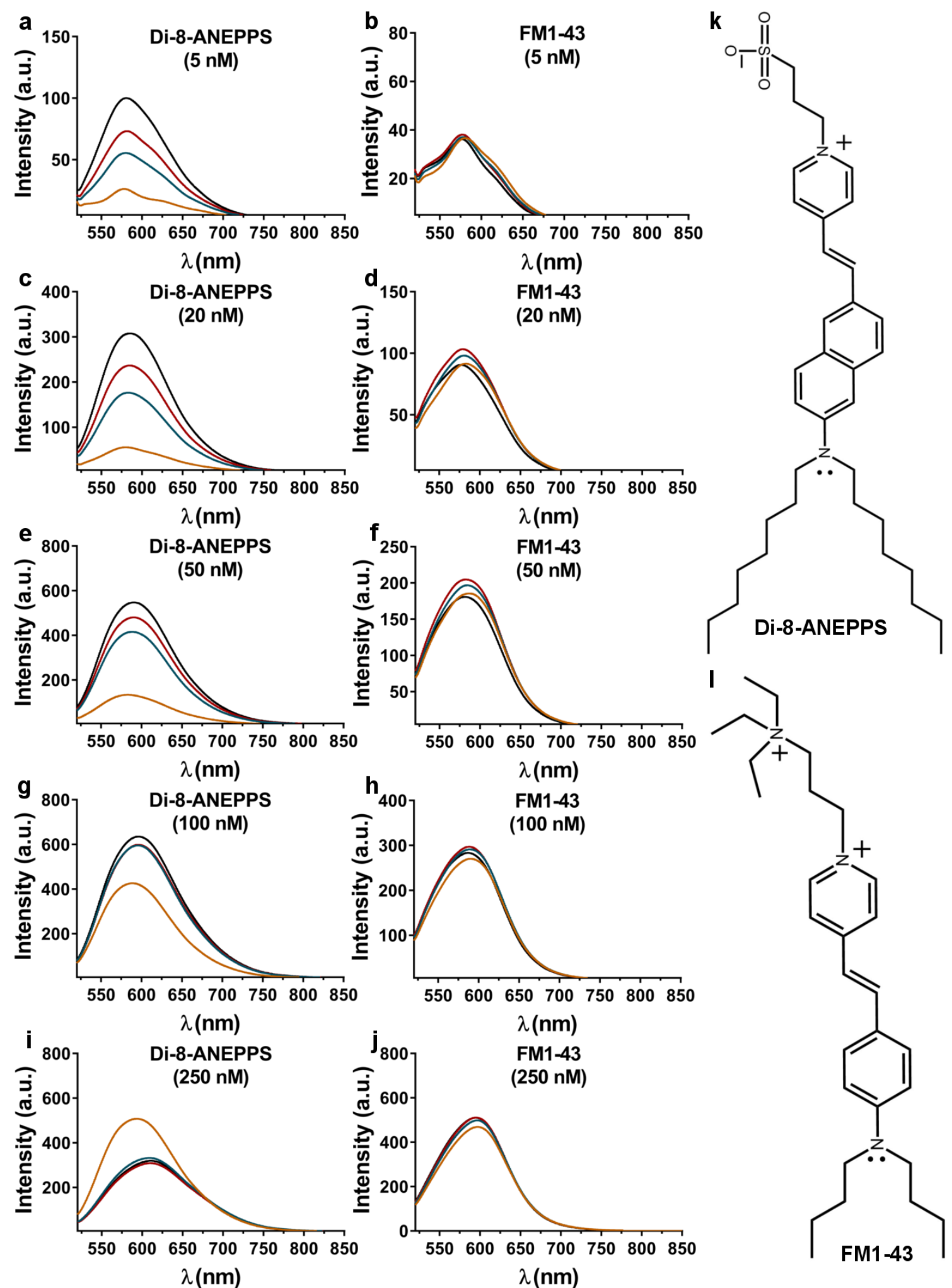

Figure S1. Bulk emission of pyridinium-like membrane dyes. a-j, Bulk emission $\left(\lambda_{\text {ex }}=488 \mathrm{~nm}\right)$ from $130 \mathrm{~nm}$ liposomes stained with different concentrations of Di-8-ANEPPS and FM1-43 at 0 (orange), 60 (blue), 120 (red), and 270 minutes (black). k, Molecular structure of Di-8-ANEPPS. l, Molecular structure of FM1-43. 

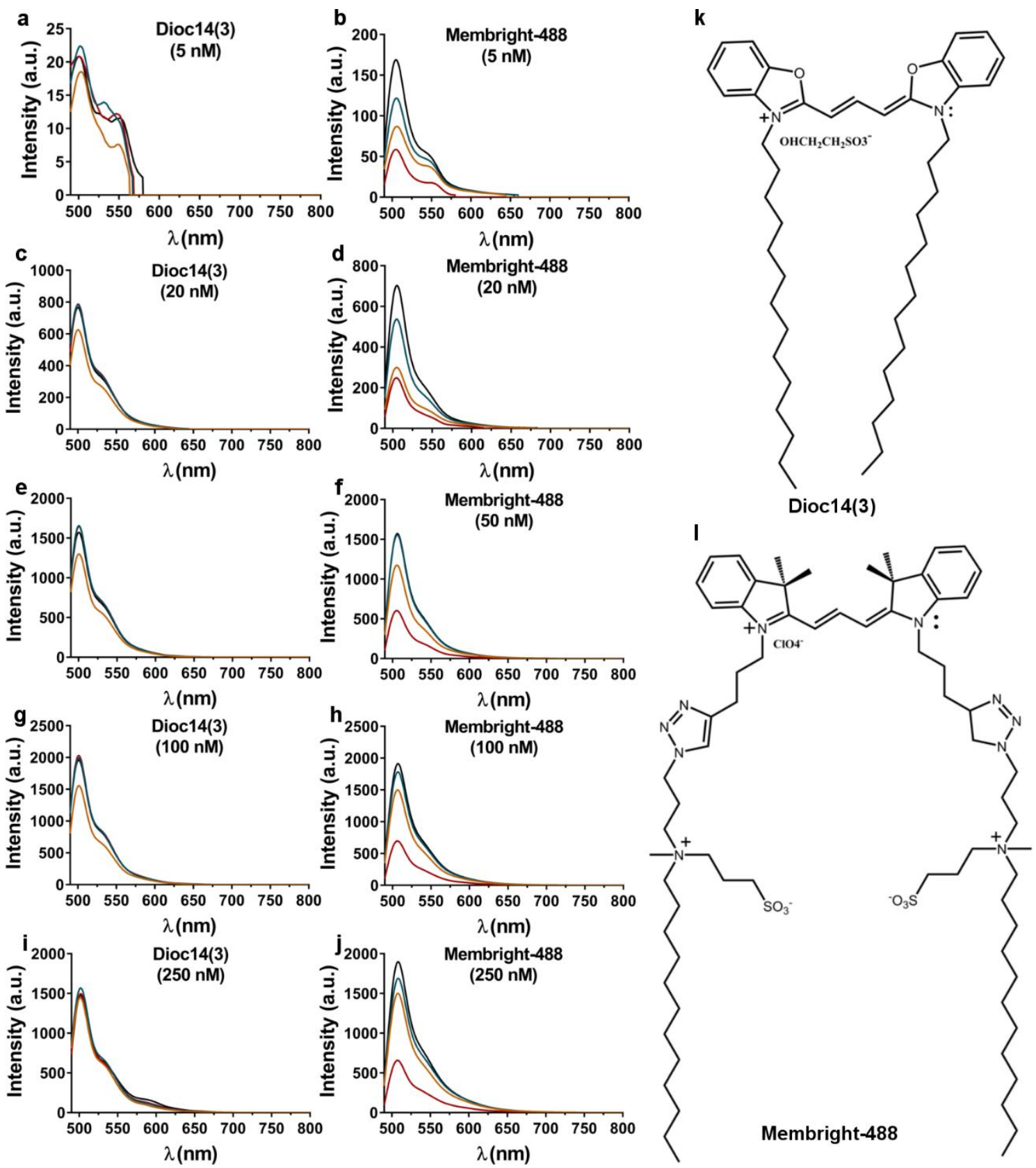

Figure S2. Bulk emission of dialkylcarbocyanine membrane dyes. a-j, Bulk emission $\left(\lambda_{\mathrm{ex}}=470\right.$ $\mathrm{nm}$ ) from $130 \mathrm{~nm}$ liposomes stained with different concentrations of Dioc14(3) and Membright-488 at 0 (orange), 60 (blue), 120 (red), and 270 minutes (black). k, Molecular structure of Dioc14(3). l, Molecular structure of Membright-488.

With an increase in dye concentration, the emission peak shifted to a longer wavelength, suggesting a change in the dye environment due to overcrowding of dyes on the vesicle surfaces. ${ }^{1}$ Whereas the pyridinium derivatives, Di-8-ANEPPS and FM1-43, intercalate entirely within the lipid bilayer, ${ }^{2}$ the dialkylcarbocyanine membrane dyes, Dioc14(3) and Membright-488, use hydrocarbon side chains to anchor into the vesicle surface. ${ }^{3}$ 

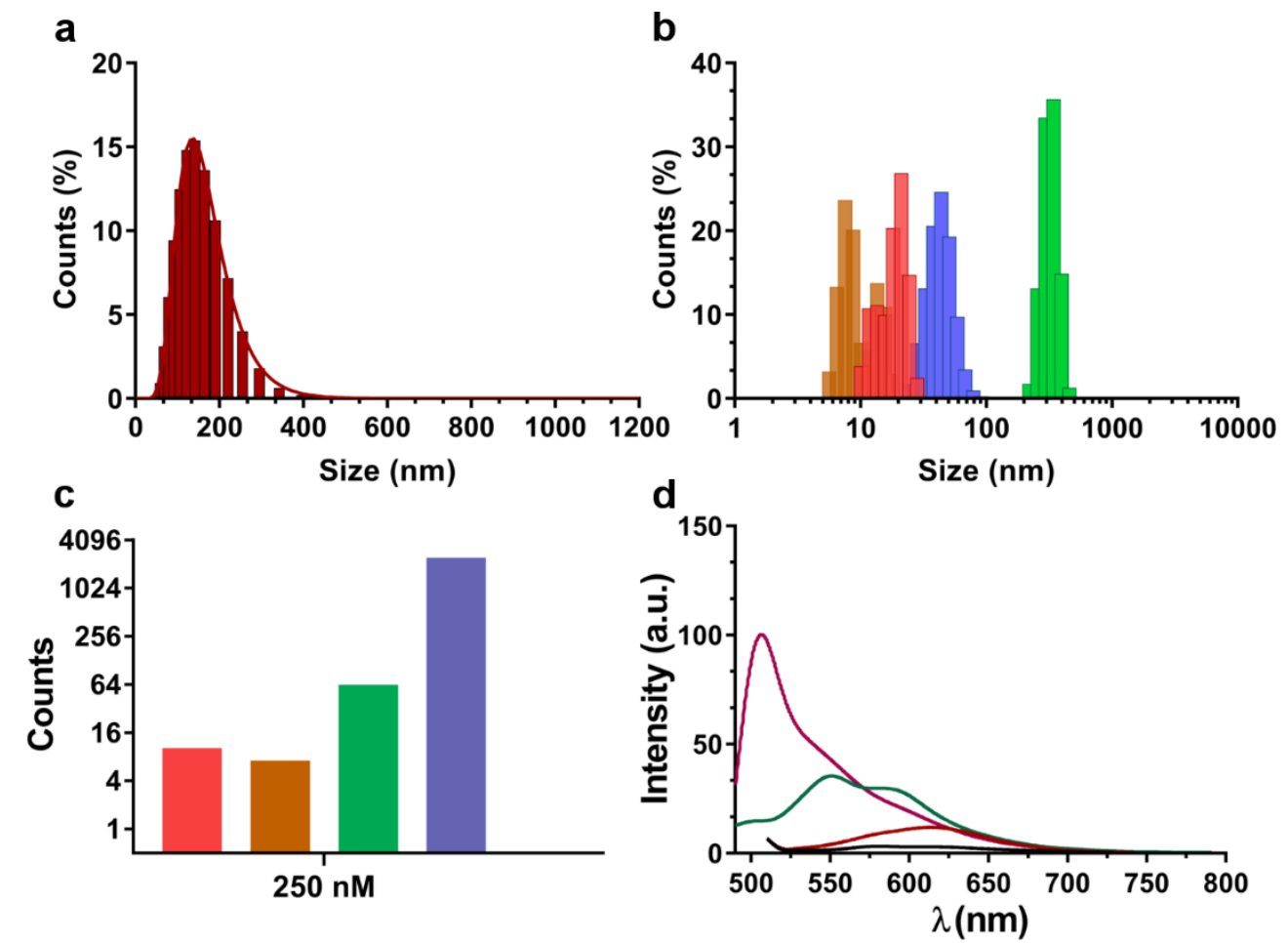

Figure S3. Aggregation of membrane dyes in buffer. a, DLS analysis of B-lipo (centered at $\sim 130 \mathrm{~nm}$ ) in 0.1X PBS. b, DLS analysis of membrane dyes in buffer (250 nM final concentration). The size distributions correspond to FM1-43 (orange), Di-8ANEPPS (pink), Membright-488 (blue), and Dioc14(3) (green). c, Number of particles counted in flow for Di-8-ANEPPS (pink), FM1-43 (orange), Dioc14(3) (green), and Membright-488 (blue) at a final concentration of $250 \mathrm{nM}$ during three minutes of acquisition. d, Bulk emission of Di-8-ANEPPS (black), FM1-43 (red), Dioc14(3) (green), and Membright-488 (violet) in buffer without liposomes (dye concentration, $250 \mathrm{nM}$ ). Comparison of the emission peaks from dye in buffer and from dye in liposomes (dye concentration $50 \mathrm{nM}$ ) after $1 \mathrm{~h}$ of equilibration; we observed an increase in emission intensity of 20-fold for Di-8-ANEPPS, 2-fold for FM1-43,7-fold for Dioc14(3), and 2fold for Membright-488. 

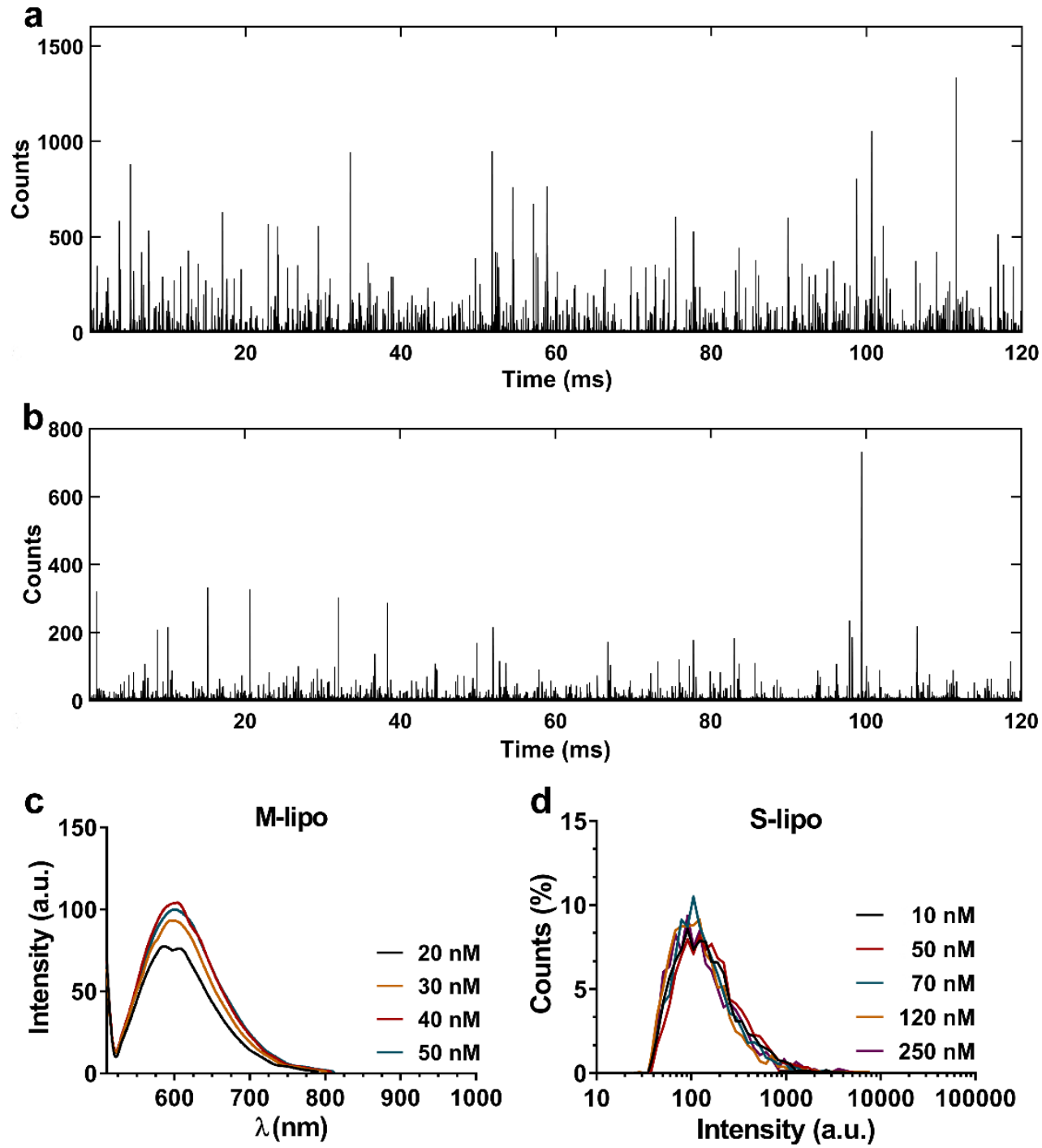

Figure S4. Optimization of staining of liposomes. a, Segment of the flow trajectory of M-lipo stained with $40 \mathrm{nM}$ Di-8-ANEPPS. b, Segment of the flow trajectory of S-lipo stained with $50 \mathrm{nM}$ Di-8-ANEPPS. c, Bulk emission from M-lipo stained with different concentrations of Di-8-ANEPPS after $2 \mathrm{~h}$ of equilibration. d, Intensity distributions from flow analysis for S-lipo stained with different concentrations of Di-8-ANEPPS. The intensity distribution was centered at 122 (10 nM dye), 136 (50 nM dye), 113 (70 nM dye), 104 (120 nM dye), and 108 (250 nM dye). 

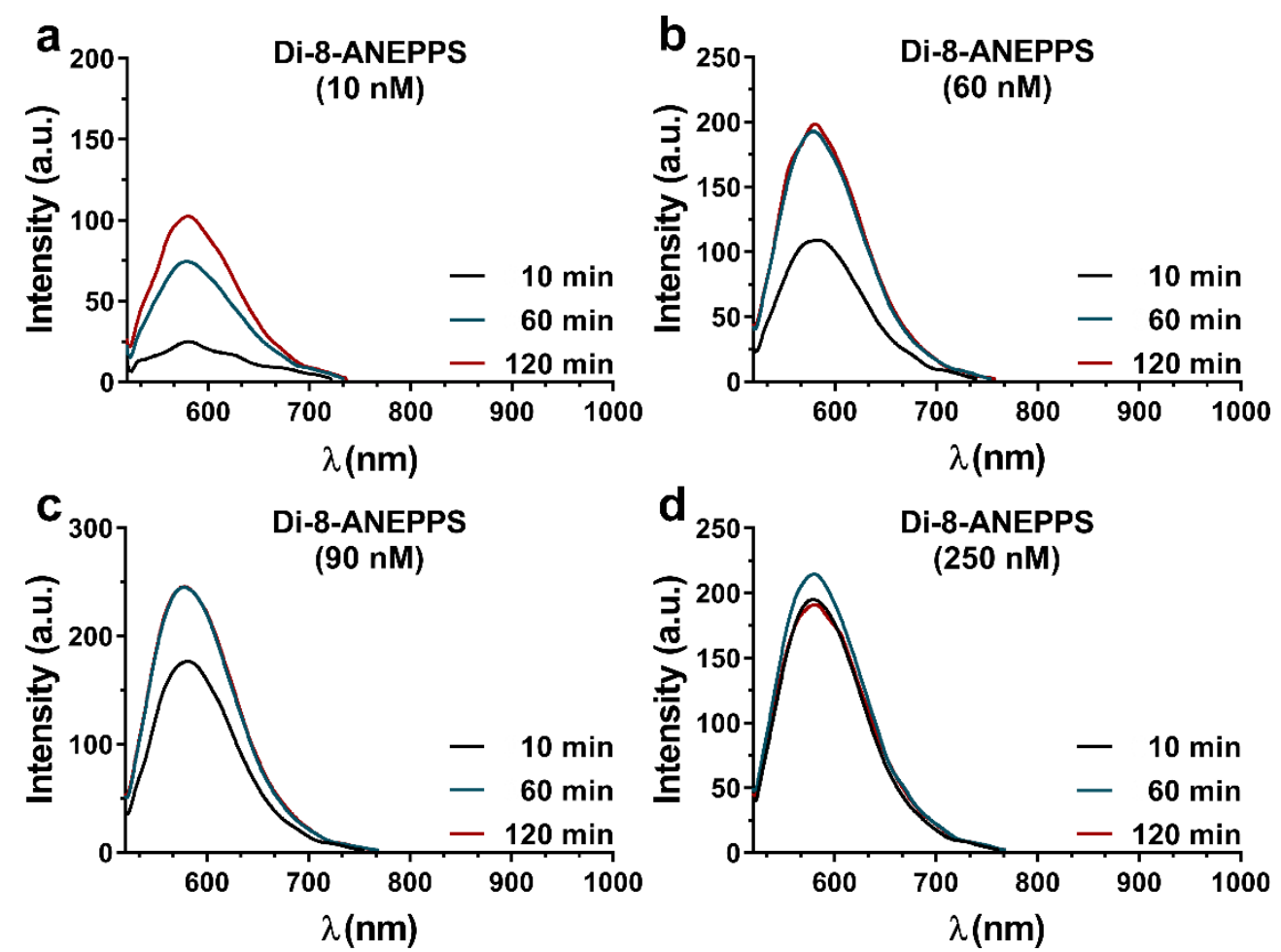

Figure S5. Bulk emission of SEs stained with Di-8-ANEPPS. a-d, Bulk emission of SEs stained with different concentrations of Di-8-ANEPPS, acquired at different times. For the $10 \mathrm{nM}$ sample, the dye concentration was not high enough to saturate the vesicles, as indicated by the continued increase in peak intensity. In contrast, the emission intensity decreased over time for $250 \mathrm{nM}$ samples, indicating quenching of dye fluorescence due to overcrowding on the vesicle surfaces.

\section{Optimization of SEs staining with Di-8-ANEPPS}

The optimization of staining conditions was achieved by systematically screening the following parameters:

1. Equilibration time: $6 \mu \mathrm{L}$ of SE stock solution $\left(2.85 \times 10^{11}\right.$ particles $\left./ \mathrm{mL}\right)$ was diluted in $1 \mathrm{~mL}$ of buffer (PBS-1X, pH 7.4) and stained with $10 \mu \mathrm{L}$ of a 1, 6, 9, or $25 \mu \mathrm{M}$ Di-8-ANEPPS solution. The bulk emission of each sample was measured over time (see Fig. S5), and a minimum time of $2 \mathrm{~h}$ before flow analysis was selected.

2. Concentration of dye given a fixed SE concentration: $6 \mu \mathrm{L}$ from SE stock solution was diluted in $1 \mathrm{~mL}$ of buffer and stained with $10 \mu \mathrm{L}$ of a $0.5,3,5,6,10$, or $25 \mu \mathrm{M}$ Di-8-ANEPPS solution.

3. Concentration of SE given a fixed dye concentration: Starting from an initial concentration of $2.85 \times 10^{11} \mathrm{SE} / \mathrm{mL}$, a volume of $50,20,10$, or $2 \mu \mathrm{L}$ was diluted in $1 \mathrm{~mL}$ buffer and stained with $10 \mu \mathrm{L}$ of $6 \mu \mathrm{M}$ dye solution.

4. EtOH concentration ( $/ \mathrm{v} \%$ ): $10 \mu \mathrm{L}$ of SE stock solution was diluted in $1 \mathrm{~mL}$ of buffer and stained with the same concentration of dye $\left(3.61 \times 10^{13}\right.$ particles per $1 \mathrm{~mL}$ of final volume). The volume and 
concentration of dye solution added to SEs was adjusted to give a final concentration of EtOH $(\mathrm{v} / \mathrm{v})$ equal to $0.1,0.5,1,2$, or $3 \%$.

5. Reaction volume for SE staining: SEs $\left(2.85 \times 10^{11}\right.$ particles $\left./ \mathrm{mL}\right)$ were diluted 100 -fold in buffer and aliquots of $1,0.5$, and $0.02 \mathrm{~mL}$ were stained with 10,5 , and $0.2 \mu \mathrm{L}$ of $6 \mu \mathrm{M}$ dye solution, respectively.

6. Dilution of both dye and SEs given a constant dye/vesicle ratio: A volume of 6, 3, 1, 0.6, or $0.1 \mu \mathrm{L}$ of SE stock solution was diluted in $1 \mathrm{~mL}$ buffer and stained with $10 \mu \mathrm{L}$ of dye in EtOH. The concentration of Di-8-ANEPPS was adjusted to maintain a constant ratio of $2.11 \times 10^{4}$ dyes per vesicle.

\section{General strategy for EVs staining using Di-8-ANEPPS}

Based on these results, a practical strategy for defining optimal staining conditions is as follows: (a) Identify the upper limit of the dye/vesicle ratio for a given EV concentration by increasing the amount of dye until bulk emission at time zero versus dye concentration deviates from linearity. (b) Perform a more detailed titration of dye within this range of concentration to identify the optimal dye concentration for staining (which is the one that ensures the highest bulk emission after equilibration, before quenching of dye fluorescence). (c) Stain EVs with the optimal dye concentration and wait at least 2 hours before analysis. 
a

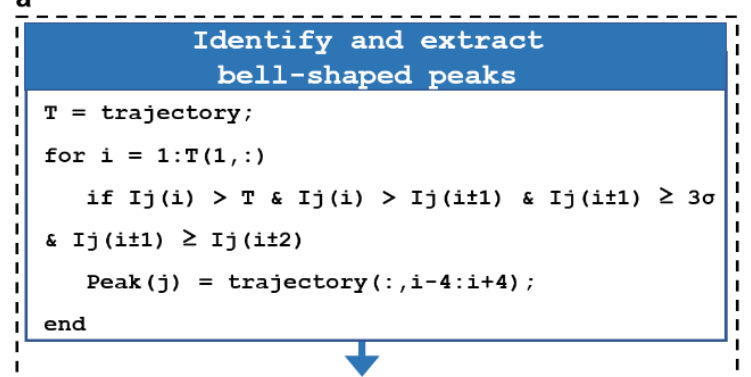

Fit of peak(j) to bell-shaped curve

$\mathrm{B}=$ baseline

for $j=1: n$

gaussian $=A \star \exp \left(-\left((t-\mu)^{\wedge} 2\right) /\left(2 \star \sigma^{\wedge} 2\right)\right)+B$;

$\mathrm{t}=1: 9$;

A $=$ Amplitude_initial-guess;

$\mu=$ Center_initial_guess ;

$\sigma=$ sigma_initial_guess ;

$\mathrm{x} 0=[\mathrm{x}(1) \mathrm{x}(2) \mathrm{x}(3)]$;

Gauss $(j)=\operatorname{nlinfit}\left(t, \operatorname{Peak}(j), \operatorname{gaussian}, x_{0}\right)$;

$\mathrm{PT}(j)=6 \sigma(j)$

end

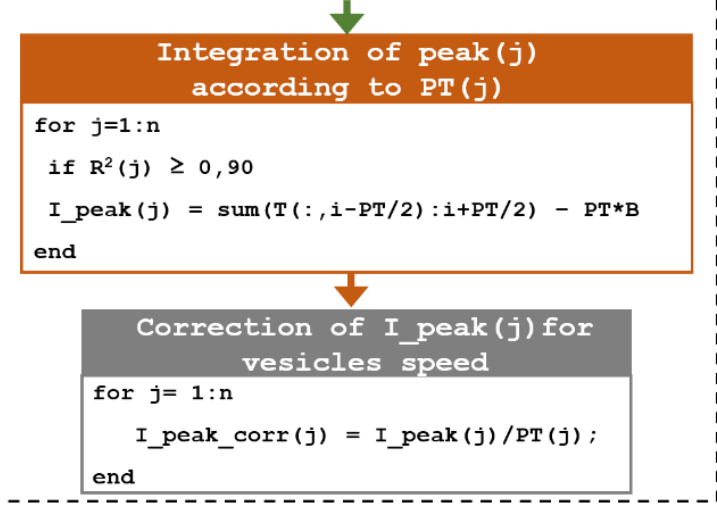

b
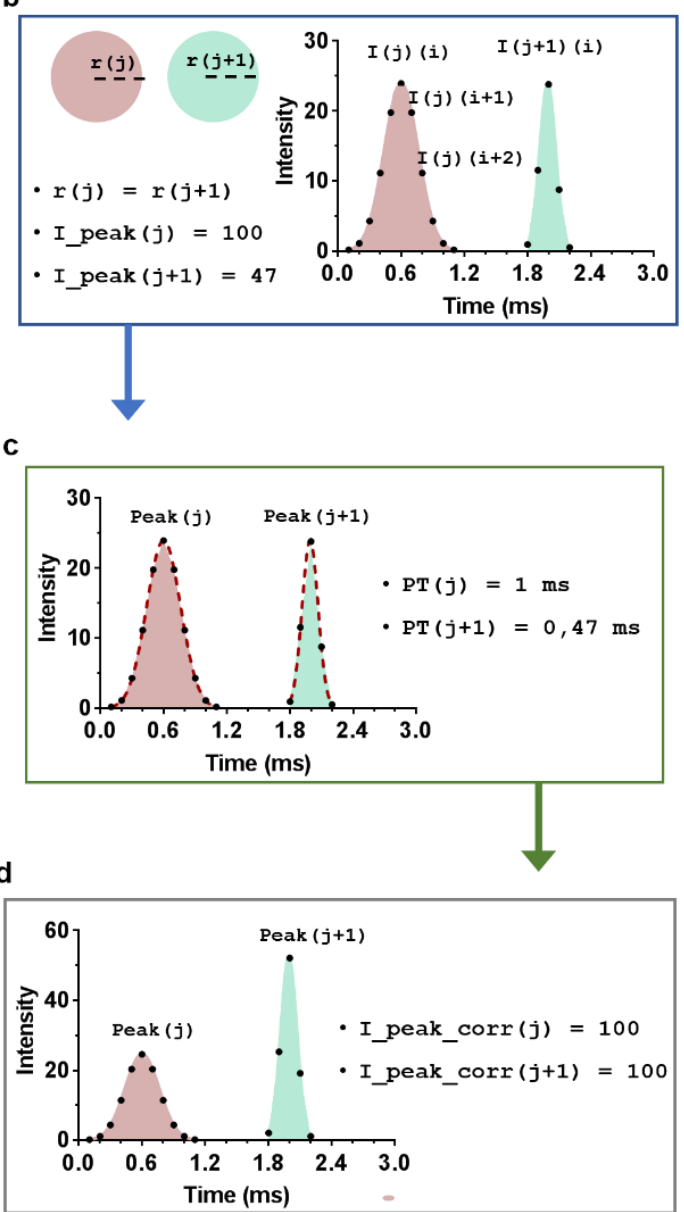

Figure S6. Pseudo code for analysis of SE sizes in flow. a, Flowchart describing the pseudo code used to analyze the SE sizes. The first step (blue) corresponds to the identification and isolation of those peaks that possess a bell-like shape and, therefore, can be analyzed to extract the SE passing time. The second step (green) represents the fitting of each individual peak to a Gaussian-like curve

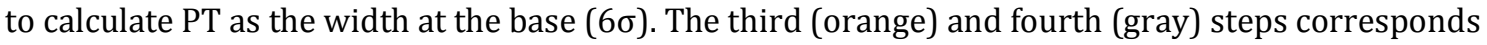
to the integration of each peak according to its width and the correction by the flow component, respectively. b-d, Representation of a hypothetical scenario in which two EVs with the same size flow at different speeds ( $1 \mathrm{~ms}$ and $0.47 \mathrm{~ms}$ PT). Without correction from the flow component, the integrated signal (area) would have two different values, producing two different size estimates. Instead, by fitting to a Gaussian curve (c), the PT of each vesicle is calculated to correct the corresponding integrated signal (d). 


\section{Uncertainty in EV size estimates}

A) Theory. The integrated fluorescence intensity $\left(I_{e}\right)$ of a vesicle flowing inside the microfluidic channel (flow along positive $\mathrm{x}$-direction, optical axis along the z-direction and $\mathrm{y}$ axis is horizontal and perpendicular to the direction of flow) can be conceptualized as follow:

$$
I_{e}=f\left(P T, F_{H}(y) F_{V}(z), n_{d}(r)\right)
$$

According to Eq. S1, $I_{e}$ can be thought as a function of:

- $P T$, the time a vesicle spends to cross the sample volume. As described in the manuscript, we divided each $I_{e}$ by the corresponding estimated passing time $\left(P T_{e}\right)$ to obtain a normalized integrated intensity $\left(I_{N}\right.$, see Eq. 5) which is independent of PT;

- $F_{H}(y) F_{V}(z)$, distributions of the instrument function along $y$ and $z$ directions. Both the intensity of the laser illumination and the efficiency with which any generated photons are detected depend on the trajectory taken by the vesicle when crossing the sample volume. Given the relatively small passing times and the low diffusion coefficient of membranous vesicles $\left(\sim 10^{-8} \mathrm{~cm}^{2} \mathrm{~s}^{-1}\right),{ }^{4}$ we can assume that each vesicle flows across the sample volume following a straight trajectory (with constant $y$ and $z$ and no lateral diffusion), and therefore $F_{H}(y)$ and $F_{V}(z)$ can be considered as two separated factors. Thus, the measured intensity $I_{e}$ is proportional to the product $F_{H}(y) F_{V}(z)$ which, in turn, is proportional to the integral along the $x$-axis of the instrument function for a specific set of $y$ and $z$. If we knew the values of $y$ and $z$ for a particular vesicle as it traverses the sample volume, we could divide $I_{e}$ by $F_{H}(y) F_{V}(z)$ just as we do with $P T_{e}$. However, since we cannot determine the vesicle coordinates along $y$ and $z$, these unknown multipliers introduce additional errors, to account for in the final uncertainty of normalized intensity $u\left(I_{N}\right)$.

- $n_{d}(r)$, number of dye molecules. This is proportional to the vesicle's radius $r$, and is Poisson distributed around the average number of dyes-per-vesicle.

Each source of uncertainty was estimated via complementary experiments (see below), and then combined according to Eq. S2: ${ }^{5}$

$$
u\left(I_{N}\right)=I_{N} \cdot \sqrt{\left(\frac{u\left(I_{e}\right)}{I_{e}}\right)^{2}+\left(\frac{u\left(n_{d}\right)}{\bar{n}_{d}}\right)^{2}+\left(\frac{u\left(F_{H}\right)}{\bar{F}_{H}}\right)^{2}+\left(\frac{u\left(F_{V}\right)}{\bar{F}_{V}}\right)^{2}+\left(\frac{u\left(P T_{e}\right)}{P T_{e}}\right)^{2}}
$$

Finally, the percentage uncertainty in size estimate $(u(s) \%)$ was derived according to Eq. S3-S5, where $s$ is the vesicle size estimated from flow and $\alpha$ is the intensity-to-size conversion factor (scaling factor):

$$
s=2 \alpha \sqrt{\frac{I_{N}}{4 \pi}}
$$




$$
\begin{gathered}
u(s)=\sqrt{\frac{\alpha^{2}}{4 \pi I_{N}} u^{2}\left(I_{N}\right)+\frac{I_{N}}{\pi} u^{2}(\alpha)} \\
u(s) \%=\frac{u(s)}{s} \cdot 100
\end{gathered}
$$

B) Calculation of the scaling factors $\alpha$ and the corresponding uncertainties. By fitting the size distribution from Cryo-EM data to a log-normal, we estimated its center (as the median $\mathrm{M}$ of distribution) and the corresponding 95\% confidence intervals $( \pm m)$. The scaling factor $\alpha$ was that number that allowed us to center the size distribution derived from flow data (according to Eq. S3) at M. whereas $\alpha_{\max }$ and $\alpha_{\min }$ represented those values required to center the size distribution from flow at $+m$ and $-m$. respectively. Therefore. $\alpha_{\max }$ and $\alpha_{\min }$ can be thought as the upper and lower bounds of uncertainty upon $\alpha$. and the confidence intervals $u(\alpha)$ can be estimated as $\frac{\alpha_{\max }-\alpha_{\min }}{2}$.

C) Estimation of integrated fluorescence intensity and its uncertainty. Ie was measured according to Eq. 2 (see manuscript), whereas the uncertainty $u\left(I_{e}\right)$ was calculated according to Eq. S6:

$$
u\left(I_{e}\right)=\sqrt{\sum_{i=1}^{n} I_{i}+n u(b)^{2}}
$$

Eq. S6

$I_{i}$ is the intensity in the $i$ th time bin, $b$ is the baseline (median of intensity values from the whole trajectory) and $n$ are the number of points in time when the vesicle is in the sample volume (equal to the base width of the peak).

D) Estimation of passing time and its uncertainty. $P T_{e}$ and $u\left(P T_{e}\right)$ were estimated from fitting each vesicle peak to a gaussian-like function. Namely, $P T_{e}$ was calculated as six times the peak width $(\sigma)$ and $u\left(P T_{e}\right)$ obtained from the confidence intervals on $P T_{e}$ (i.e. six times the $95 \%$ confidence intervals of $\sigma$, obtained from the fitting).

E) Estimation of number of Di-8-ANEPPS dye-per-vesicle and its uncertainty. $n_{d}$ and $u\left(n_{d}\right)$ were estimated by performing single-molecule photobleaching experiments and cross-correlation flow analysis of SEs. To avoid interference from potential biological contaminants (e.g. cell debris, nonexosomes vesicles etc.) we focused only on those vesicles which were positive to the surface protein Tetraspanin CD63 (CD63+-SEs). The latter is a specific biomarker for SEs. ${ }^{6}$ Thus, after staining SEs with Di-8-ANEPPS and incubating with an Alexa 647-anti-CD63 antibody, we performed colocalization analysis in flow to extract the sizes of CD63+-SEs (see Fig. S7). In separate experiments using Total Internal Reflection Fluorescence microscopy (TIRF), we measured the emission intensity of individual Di-8-ANEPPS dyes by single-molecule bleaching (see Fig. S8), and measured the total emission of CD63+SEs. CD63+ SE emission was converted to vesicle size by comparison with the subpopulation of colocalized SEs from flow (see Fig. S8). The number of dyes-per- vesicle was estimated by dividing the total emission of CD63+-SEs by the emission intensity of a single dye molecule (see below). 

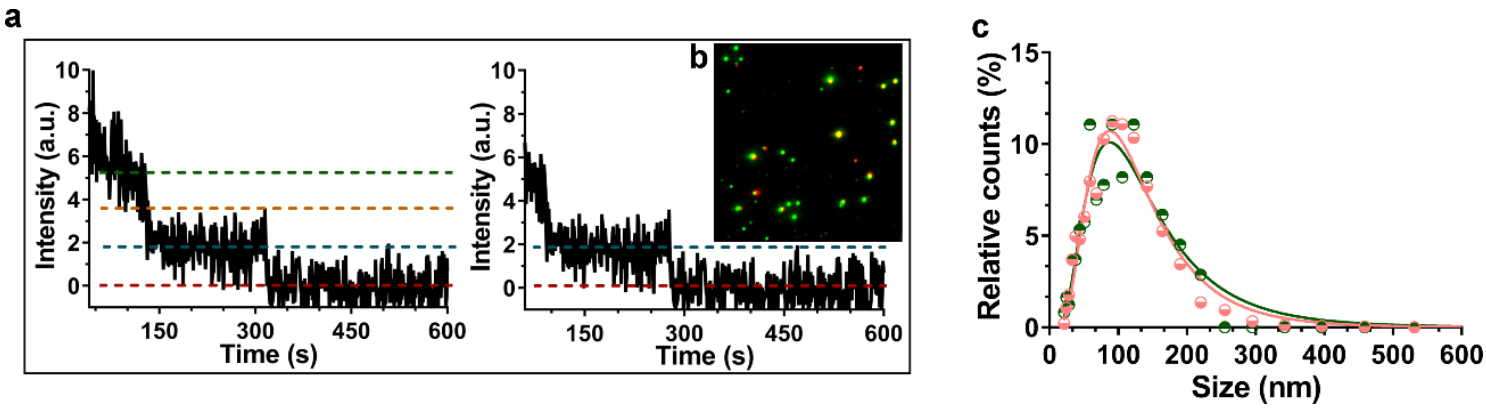

Figure S7. TIRF and Flow analysis for $\boldsymbol{n}_{\boldsymbol{d}}$ estimation. a, Intensity traces from single-molecule bleaching step (step $=1.84 \pm 0.22 ; \mathrm{n}=7$ measurements). b, TIRF microscopy image merging 488-nm (green) and 640-nm (red) channels, showing the overlap of Di-8-ANEPPS (green) and CD63+ (red) staining. c, Overlap of size distributions of CD63+-SEs estimated using TIRF microscopy (green) and flow analysis (pink). Size distributions of colocalized vesicles from flow data and TIRF experiment were centered (median \pm SD) at $87.5 \pm 50.7$ and $87.5 \pm 55.2$, respectively, and by analyzing 245 (in TIRF experiment) and 2800 (in flow experiment) vesicles.

Experimental procedure (Flow). For flow experiments, we stained SEs using the previously optimized conditions, namely $6 \mu \mathrm{L}$ of SE stock solution $\left(2.85 \times 10^{11}\right.$ particles $\left./ \mathrm{mL}\right)$ was diluted in $1 \mathrm{~mL}$ of buffer (PBS-1X, pH 7.4) and stained with $10 \mu \mathrm{L}$ of a $6 \mu \mathrm{M}$ Di-8-ANEPPS solution (fully labeled exosomes). Flow analysis was performed after $2 \mathrm{~h}$ of equilibration, and the sizes of the CD63-labeled vesicles were extracted from the total population of SEs by analyzing only the events in the $488 \mathrm{~nm}$ channel that also produced a colocalized signal in the $640 \mathrm{~nm}$ channel (see Fig. S7c and S8). Correlation analysis was performed using the MATLAB xcorr function.

Experimental procedure (TIRF). For TIRF experiments, glass coverslips were cleaned sequentially with acetone, $\mathrm{KOH}$, and methanol under sonication. After each step, the coverslips were washed with Milli-Q water twice and dried using nitrogen flow. The molded PDMS with 5-mm wells was rinsed with Milli-Q water and EtOH several times and dried using nitrogen flow. Following treatment with oxygen plasma cleaner, the molded PDMS and coverslips were assembled to produce the final chip for imaging. SEs $\left(6 \mu \mathrm{L}\right.$ from a solution of $2.85 \times 10^{11}$ particles $\left./ \mathrm{mL}\right)$ were incubated with Alexa 647 anti-human CD63 antibody ( $1 \mu \mathrm{L}$ of $50 \mu \mathrm{g} / \mathrm{mL}$ solution; BioLegend, Inc.) for $1 \mathrm{~h}$, then labeled with Di-8-ANEPPS. After $2 \mathrm{~h}$, the stained SEs were purified using an iZON-70nm column to remove free antibody, and 10-20 $\mu \mathrm{L}$ of SE solution was loaded into a chip for analysis.

Two samples were prepared by staining the same SE concentration with $10 \mu \mathrm{L}$ of $6 \mu \mathrm{M}$ or $5 \mathrm{nM}$ dye solution in EtOH. The first sample (fully labeled exosomes, to ensure saturation of vesicles) was analyzed to calculate vesicle sizes by acquiring the total fluorescence intensity from each EV; the second sample (sparsely labeled exosomes, to ensure $\sim 1$ dye-per-vesicle) was used to measure the intensity relative to a single dye molecule using photobleaching experiments. The latter experiment required the addition of 
oxygen scavengers (11 mM glucose, $0.8 \mathrm{mg} / \mathrm{mL}$ pyranose 2 -oxidase, and $0.2 \mathrm{mg} / \mathrm{mL}$ catalase) to the SE solution to improve dye stability. The total fluorescence intensity of each vesicle was measured using an EM-CCD camera, and was calculated as the average of 10 consecutive images (100 ms per frame) using ImageJ. The laser intensity was kept low $(10 \mathrm{~mW})$ to avoid degradation of dyes. The intensity values were transformed into sizes by comparison with data from cross-correlation flow experiments. To prevent analyzing dye aggregates or membranous debris, we focused only on CD63-positive vesicles.

Single-dye intensity was measured following the bleaching of Di-8-ANEPPS inside a vesicle over time (100 ms per frame) after the $488 \mathrm{~nm}$ laser was quickly increased from 10 to $100 \mathrm{~mW}$. Several bleaching steps were calculated from traces of individual exosomes by fitting the intensity distributions to a Gaussian function and correcting for the background. The average intensity-step was scaled back to match the data collected at $100 \mathrm{~mW}$ and used to calculate the number of dyes per exosome. 

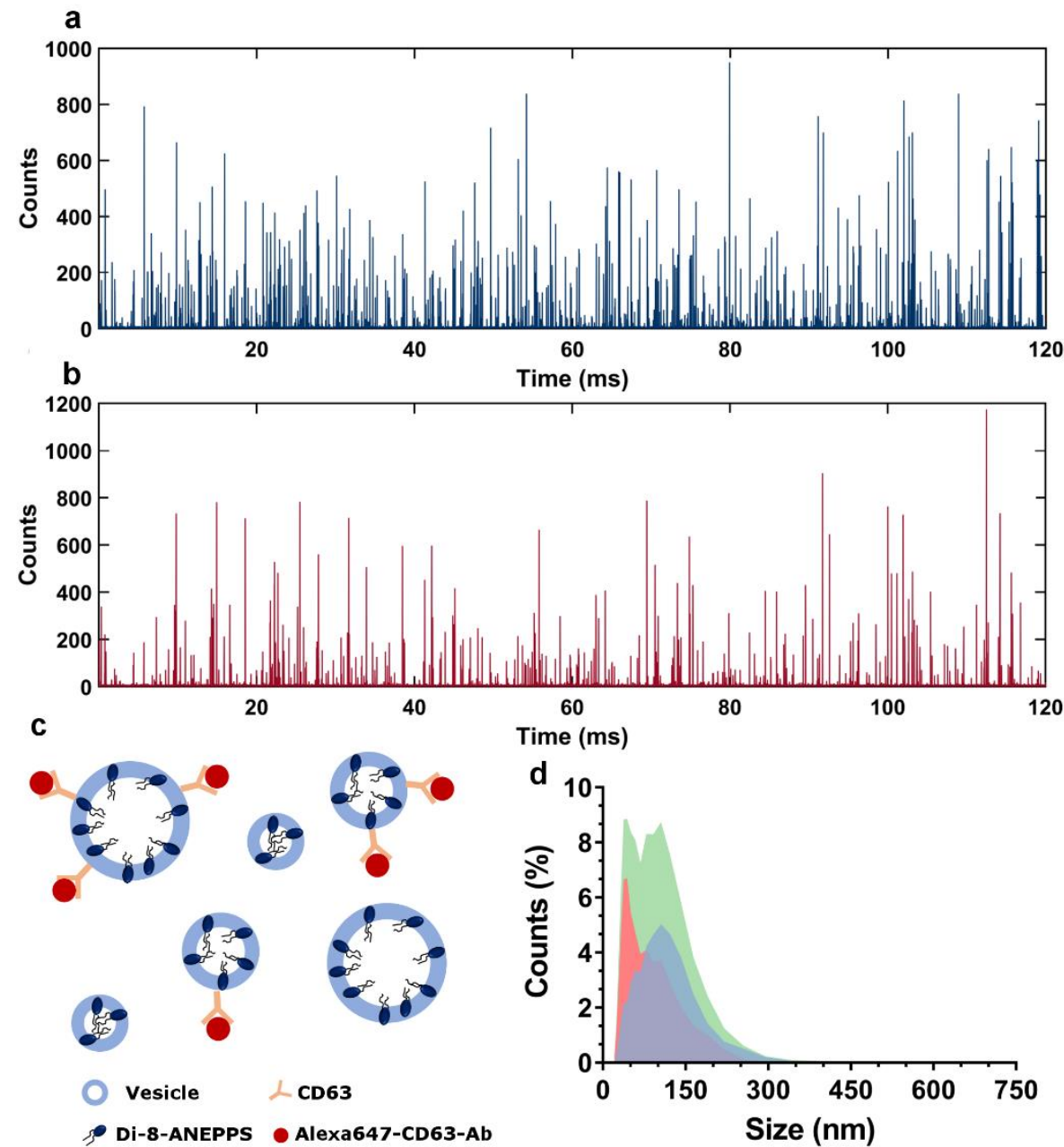

Figure S8. Cross-correlation analysis of SEs conjugated with Alexa 647CD63. a, Segment of the flow trajectory in the 488-nm channel (Di-8-ANEPPSpositive vesicles). b, Segment of the flow trajectory in the 640-nm channel (Alexa 647-CD63-positive vesicles). c, Schematic of CD63-positive and CD63negative SEs stained with Di-8-ANEPPS. d, Size distributions of total SEs (green), CD63-negative SEs (red), and CD63-positive SEs (blue). 
Uncertainty propagation. From TIRF experiments we measured the total emission of CD63+-SEs $\left(I_{T I R F}\right)$ and the emission intensity of a single dye molecule $\left(I_{S T E P}\right)$, then each $I_{T I R F}$ value transformed into size $(s)$ through the Eq. S7:

$$
s=2 \beta \sqrt{\frac{I_{T I R F}}{4 \pi}}
$$

The scaling factor $\beta$ and its uncertainty $(u(\beta))$ were calculated following the same approach for the $\alpha$ factor in Eq. S3 (i.e. iteratively by comparison with the CD63+-SEs size distribution derived from crosscorrelation experiments in flow, see Fig. S8). Then, each normalized integrated intensity value in flow $\left(I_{N}\right)$ was transformed into an equivalent intensity in TIRF ( $\left.I_{T i r-N}\right)$ (Eq. S8) and the corresponding uncertainty calculated (Eq. S10), by imposing the equivalence in Eq. S9 $(\alpha \pm u(\alpha)$ are the scaling factor and its uncertainty calculated from conversion of flow intensities into sizes). The $n_{d}$ and $u\left(n_{d}\right)$ were estimated according to Eq. S11-S12 (the additional factor $n_{d}$ in Eq. S12 accounts for the inherent Poisson distribution of dyes into vesicle). The uncertainties were combined following the error propagation theory. ${ }^{5}$

$$
\begin{gathered}
2 \beta \sqrt{\frac{I_{\text {Tirf }-N}}{4 \pi}}=2 \alpha \sqrt{\frac{I_{N}}{4 \pi}} \\
I_{\text {Tirf }-N}=\frac{\alpha^{2}}{\beta^{2}} I_{N} \\
u\left(I_{\text {Tirf }-N}\right)=\sqrt{\left(\frac{2 \alpha}{\beta^{2}} I_{N} u(\alpha)\right)^{2}+\left(-\frac{2 \alpha^{2}}{\beta^{3}} I_{N} u(\beta)\right)^{2}} \\
n_{d}=\frac{I_{\text {Tirf }-N}}{I_{\text {STEP }}} \\
u\left(n_{d}\right)=\sqrt{n_{d}^{2}\left(\left(\frac{\left.u\left(I_{\text {Tirf }-N)}\right)^{2}+\left(\frac{u\left(I_{\text {STEP }}\right)}{I_{\text {Tirf }-N}}\right)^{2}\right)+n_{d}}{I_{\text {STEP }}}\right.\right.}
\end{gathered}
$$




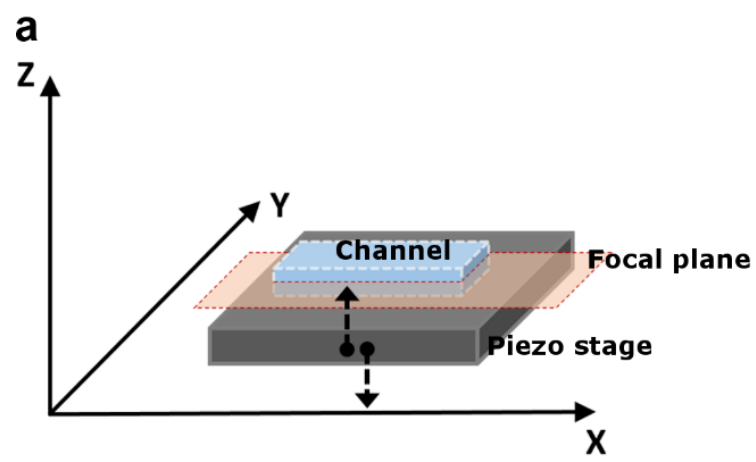

b
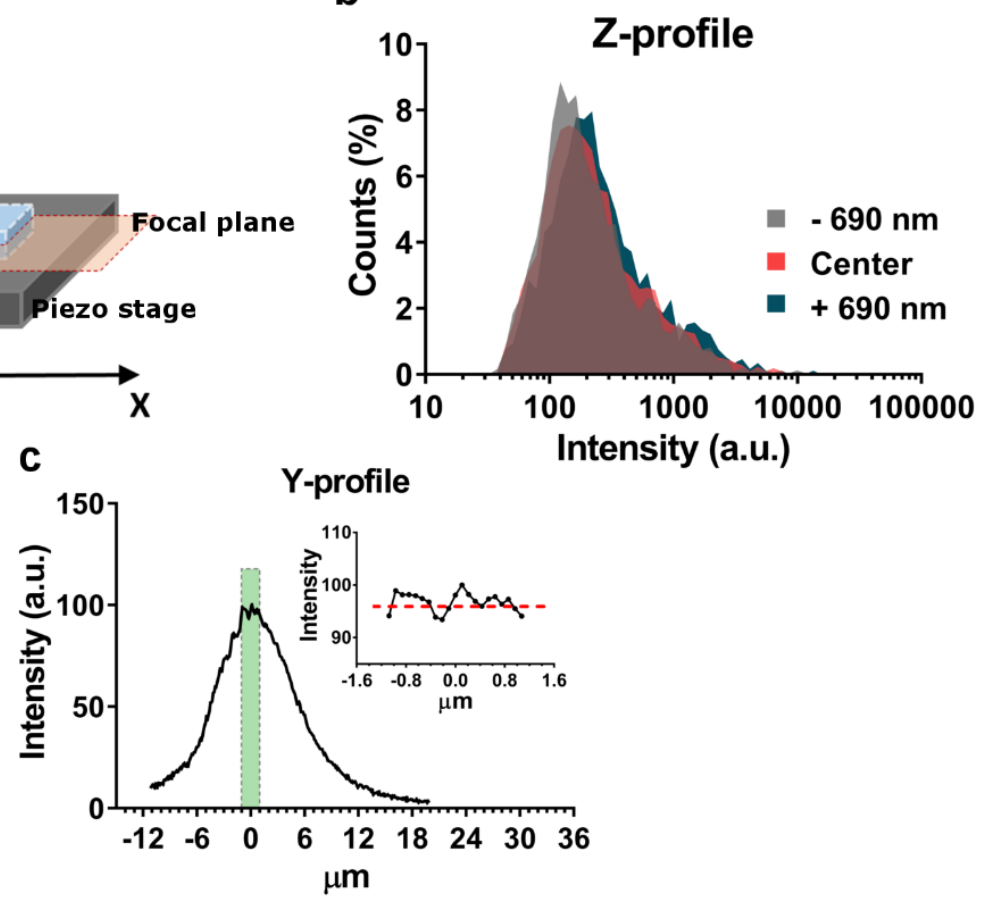

Figure S9. Variation along the $y$ - and z-directions of the instrument function. a, Schematic of the microfluidic channel on a piezo stage. The channel center was re-positioned $\sim 700 \mathrm{~nm}$ above or below the focal plane by applying either a positive or negative voltage to the piezo stage. b, Intensity distribution of SEs analyzed with the center of the channel positioned at the focal plane (pink), and at $700 \mathrm{~nm}$ above (blue) or below (gray) the focal plane. The intensity distribution was centered at 186 (gray), 206 (pink), and 237 (blue). c, Laser intensity profile along the y-direction (orthogonal to the flow direction). The insert shows the intensity (mean $=96.8 \pm 1.8$ a.u.) within the channel crosssection. (green area).

F) Estimation of variation in $\mathbf{y}$ - and z-components of the instrument function. $\bar{F}_{H}(y$-component of the instrument function) and its corresponding $u\left(F_{H}\right)$ were calculated as the mean and the standard deviation of the instrument function along y-axis within $\pm 1 \mu \mathrm{m}$ from the peak, which corresponds to the center of the microchannel (see Fig. S9c). The terms $\bar{F}_{V}$ (z-component of the instrument function) and $u\left(F_{V}\right)$ were estimated indirectly, by comparing the shift in the fluorescence intensity from SE sample (stained under optimal conditions) after repositioning the microchannel (see Fig. S9a). We began by measuring the intensity in flow with the channel in the focal plane (the position that gave maximum fluorescence intensity). Using a piezo stage with $1.33 \mu \mathrm{m} / \mathrm{V}$ transfer function and a 25 -nm step-size, we positioned the microchannel at $700 \mathrm{~nm}$ above and below the focal plane, respectively, to measure the fluorescence from SEs (see Fig. S9b). We collected $>1500$ events for a given position and compared the corresponding median intensities to calculate $\bar{F}_{V}$ and $u\left(F_{V}\right)$ as mean and standard deviation of the three measurements. 
a

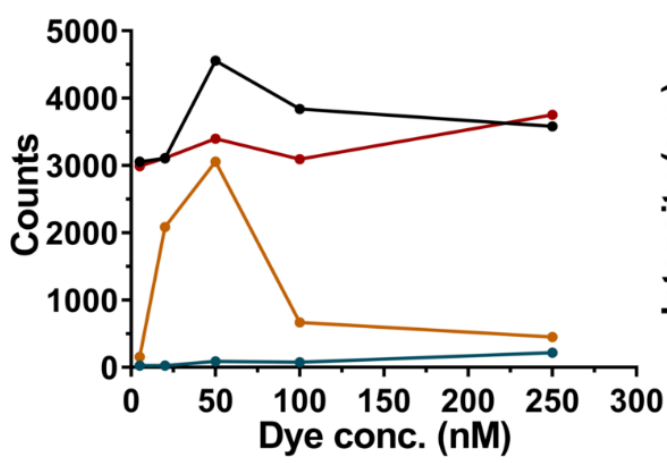

b

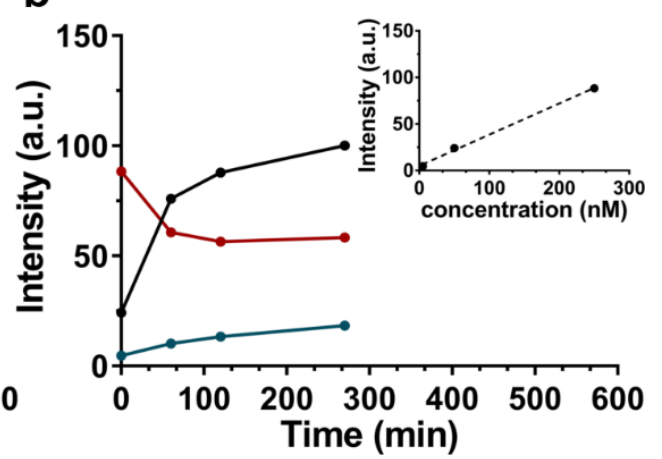

Figure S10. Selection of organic dyes for EV staining. a, Total vesicles detected in 3 min as a function of dye concentration: Di-8-ANEPPS (black), FM1-14 (blue), Dioc14(3) (orange), Membright-488 (red). b, Change in bulk emission intensity at $588 \mathrm{~nm}$ over time for different concentrations of Di-8-ANEPPS: $5 \mathrm{nM}$ (blue), $50 \mathrm{nM}$ (black), $250 \mathrm{nM}$ (red). Insert: intensity maximum at time zero as a function of Di-8-ANEPPS concentration.

Staining liposomes with Di-8-ANEPPS or Membright 488 resulted in efficient vesicle detection $(>1000$ events/min; 2000 events/min was estimated for optimal staining conditions based on a liposome concentration of $2.97 \times 10^{14}$ particles/mL (from nanoparticle tracking analysis) and a volumetric flow rate of $1 \mathrm{~nL} / \mathrm{min}$ ), even at low dye concentrations (see Fig. S10a, black and red curves). In contrast, staining with FM1-43 resulted in inefficient detection ( $<100$ events/min) even at the highest dye concentration (see Fig. S10a, blue). Staining with Dioc14(3) resulted in vesicle detection that was strongly dye concentration-dependent, with a peak at $50 \mathrm{nM}$ Dioc14(3) (see Fig. S10, orange). As shown in Fig. S10b, the bulk emission from vesicles stained with Di-8-ANEPPS either increases or decreases over time, depending on whether the concentration of dye is below or above the critical concentration (at which the quenching of fluorescence emission starts occurring). Furthermore, regardless the dye concentration, the bulk emission at time zero results directly proportional to the dye concentration itself. 

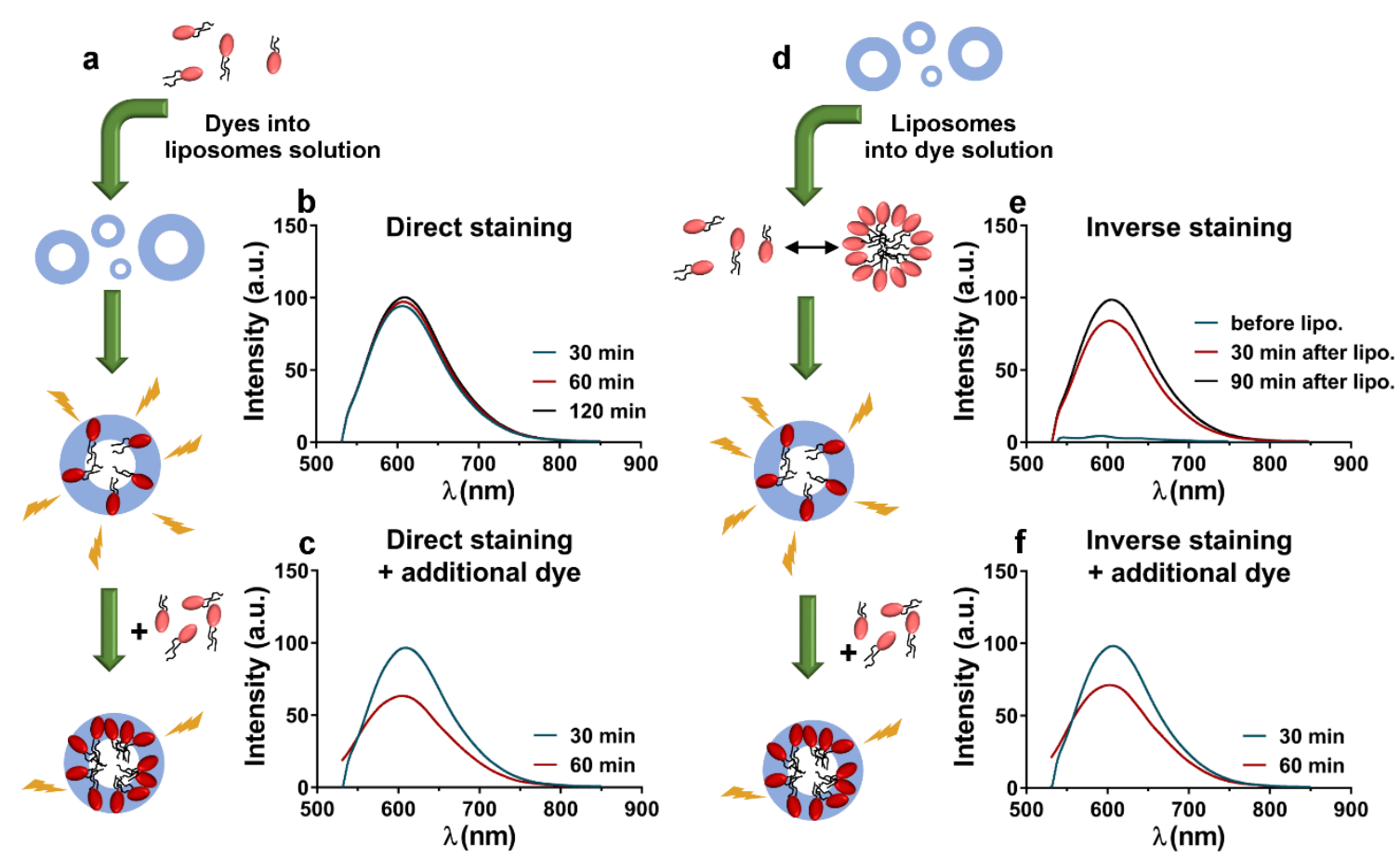

Figure S11| Equilibrium between dye molecules in vesicle membranes and in solution. a, Schematic of the staining of liposomes with Di-8-ANEPPS. b, Bulk emission $\left(\lambda_{\mathrm{ex}}=488 \mathrm{~nm}\right)$ of liposomes stained with Di-8-ANEPPS. c, Bulk emission of stained liposomes after injection of additional dye. d, Schematic of the inverse staining of liposomes with Di-8-ANEPPS. e, Bulk emission of liposomes from inverse staining. f, Bulk emission of liposomes from inverse staining after injection of additional dye

To confirm the equilibrium between dye molecules in the vesicle membrane and in solution (either as free dyes or as aggregates), we compared the bulk emission of two samples:

- Sample A (direct staining): liposomes $(130 \mathrm{~nm})$ were diluted in buffer and then Di-8-ANEPPS was added.

- Sample B (inverse staining): Di-8-ANEPPS was injected into the buffer first and then liposomes (130 $\mathrm{nm})$ were added.

For both samples, we used a final liposome concentration of $1.7 \mu \mathrm{M}$ and a final Di-8-ANEPPS concentration of $100 \mathrm{nM}$. After $2 \mathrm{~h}$ of equilibration, additional Di-8-ANEPPS was added to each sample to increase the total dye concentration to $300 \mathrm{nM}$. As shown in Fig. S11b and S11f, the bulk emission intensity reached the same plateau for both direct and inverse staining, supporting the existence of an equilibrium between dye molecules in buffer and in vesicle membranes. Furthermore, the increase in dye concentration causes an increase in the number of dye molecules intercalated into vesicle membranes, causing quenching of dye fluorescence (Fig S4 and S5). 


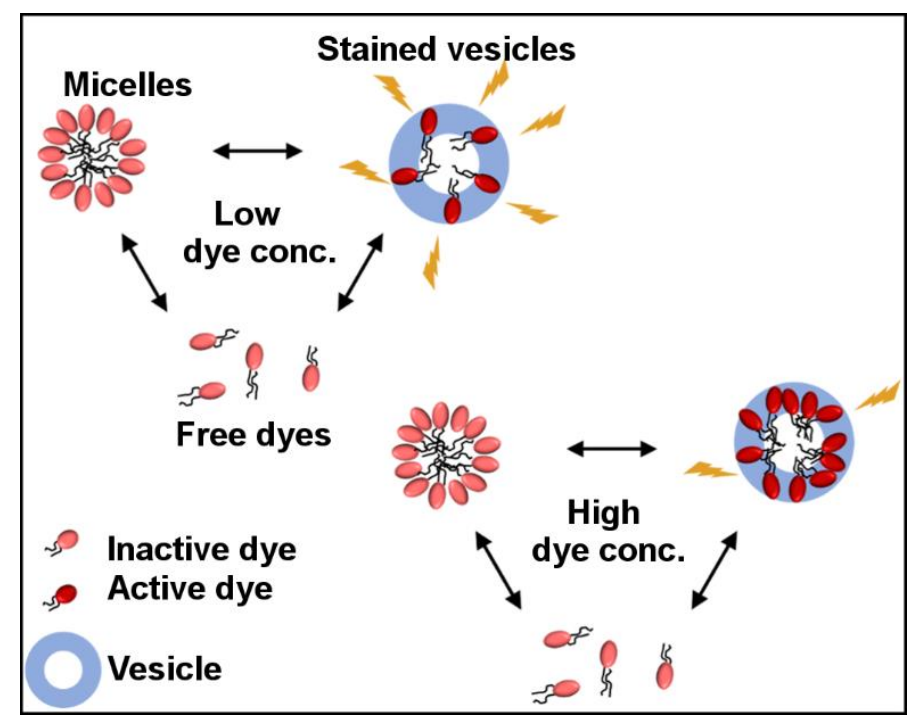

Scheme S1. Schematic of the proposed mechanism of EVs staining,m using Di-8-ANEPPS as membrane dye.

Based on our results, we propose the following mechanism of vesicle staining by Di-8-ANEPPS (see Scheme S1):

-When Di-8-ANEPPS is added to a vesicle solution, only a small fraction of the dye partitions onto vesicle surfaces ( $\sim 6 \%$ at optimal dye concentration for semen exosome (SE) samples, based on TIRF and CryoEM (see above).

- The bulk emission intensity at time zero is proportional to the dye concentration (see Fig. S10, inset), and increases or decreases over time depending on whether there is dye quenching (see Fig. S10b).

- After $\sim 2 \mathrm{~h}$, the system reaches an equilibrium state (see Fig. S11) and the bulk emission intensity reaches a plateau (see Fig. S10). 

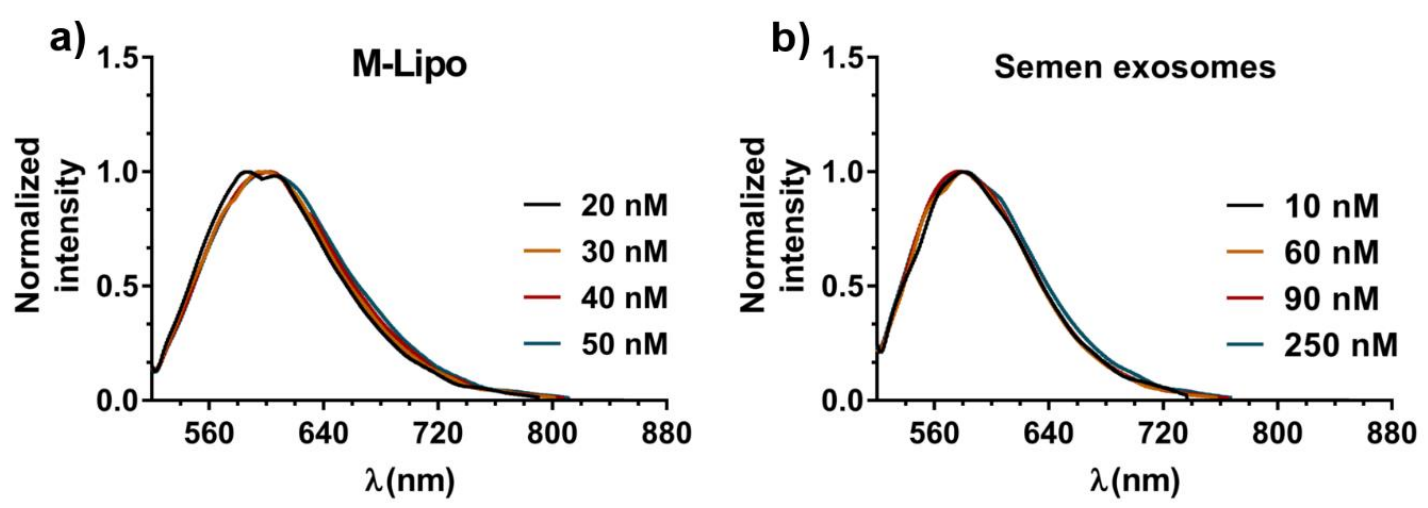

Figure S12. Bulk emission of di-8-ANEPPS at different concentration. a, Bulk emission of di8-ANEPPS $\left(\lambda_{\mathrm{ex}}=488 \mathrm{~nm}\right)$ in M-Lipo after $2 \mathrm{~h}$ of equilibration time. b, Bulk emission of di-8ANEPPS $\left(\lambda_{\mathrm{ex}}=488 \mathrm{~nm}\right)$ in semen exosomes after $2 \mathrm{~h}$ of equilibration time.

For both liposomes (Fig. S11a) and exosomes (Fig. S11b), the shift in emission spectrum of di-8-ANEPPS was negligible even at high dye concentration, when the fluorescence starts quenching (see Fig. S4c and S5). Thus, changes in local environment due to high density of dye did not affect the emission signal.

a

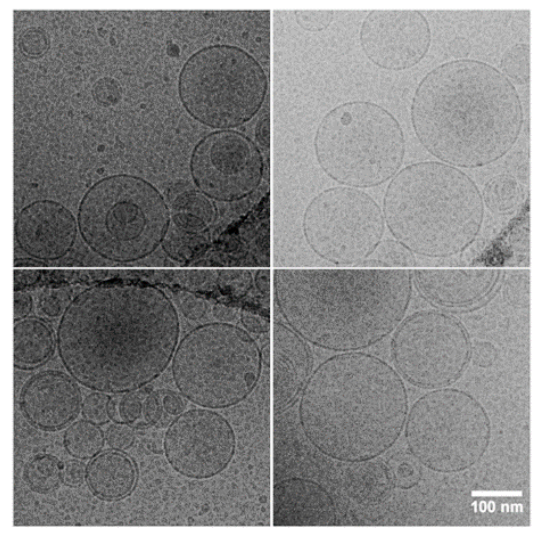

b

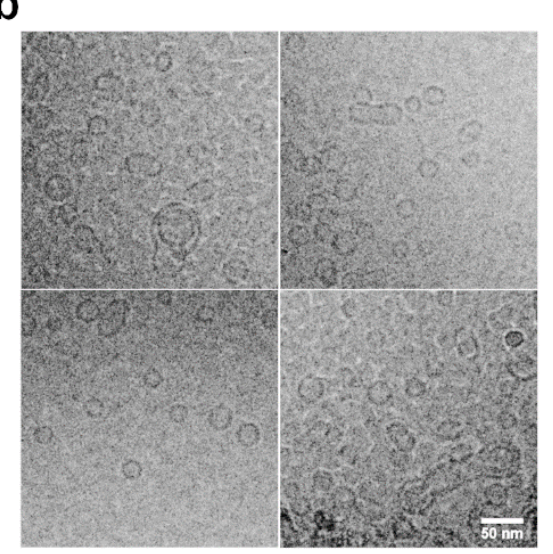

C

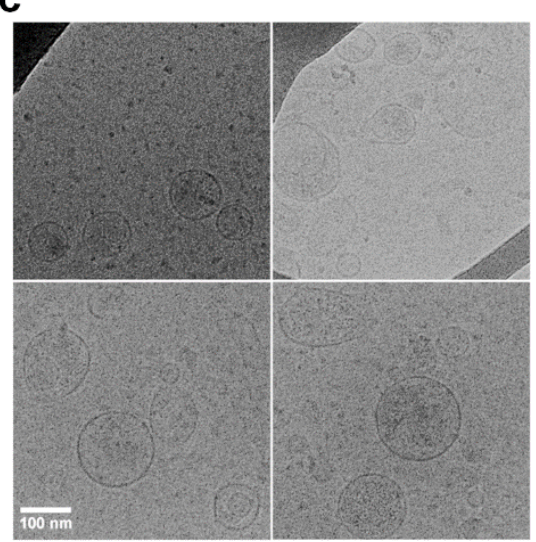

Figure S13. Cryo-EM. Representative EM images for the M-Lipo (a), S-Lipo (b) and SEs (c) samples. 
Table T1| Statistical parameters (median and SD) for the size distributions obtained during the optimization of

SEs labelling.

\begin{tabular}{|c|c|c|c|c|c|}
\hline \multicolumn{2}{|c|}{ Screening of Di-8-ANEPPS } & \multicolumn{2}{|c|}{ Screening of EtOH\% } & \multicolumn{2}{|c|}{ Screening of SEs concentration } \\
\hline $\begin{array}{l}\text { Dye/SEs } \\
\text { ratio }\end{array}$ & Median \pm SD $(\mathrm{nm})$ & $\begin{array}{c}\% \\
\text { (vol/vol) }\end{array}$ & Median \pm SD $(\mathrm{nm})$ & $\begin{array}{l}\text { Dilution } \\
\text { from C }\end{array}$ & Median \pm SD $(\mathrm{nm})$ \\
\hline $1.8 \mathrm{E}+03$ & $44.8 \pm 18.7$ & $0.1^{*}$ & $66.9 \pm 49.9$ & $1.000 x^{*}$ & $72.6 \pm 54.1$ \\
\hline $10.6 \mathrm{E}+03^{*}$ & $66.3 \pm 46.8$ & $0.5^{*}$ & $74.6 \pm 49.0$ & $0.500 \mathrm{x}$ & $61.2 \pm 44.9$ \\
\hline $17.6 \mathrm{E}+03^{*}$ & $70.7 \pm 47.0$ & $1.0^{*}$ & $71.0 \pm 46.9$ & $0.170 \mathrm{x}$ & $46.8 \pm 24.9$ \\
\hline $21.1 \mathrm{E}+03^{*}$ & $70.0 \pm 44.0$ & $2.0^{*}$ & $67.7 \pm 46.9$ & $0.100 \mathrm{x}$ & $37.3 \pm 21.5$ \\
\hline $35.2 \mathrm{E}+03^{*}$ & $71.1 \pm 42.1$ & $3.0^{*}$ & $64.2 \pm 44.3$ & $0.017 \mathrm{x}$ & $27.6 \pm 5.6$ \\
\hline $88.1 \mathrm{E}+03$ & $63.3 \pm 35.0$ & \multicolumn{2}{|c|}{$\begin{array}{c}\text { Screening or reaction } \\
\text { volume }\end{array}$} & & \\
\hline \multicolumn{2}{|c|}{ Screening of SEs } & $\begin{array}{c}\text { volume } \\
(\mathrm{mL})\end{array}$ & Median \pm SD $(\mathrm{nm})$ & & \\
\hline $\begin{array}{l}\text { Dye/SEs } \\
\text { ratio }\end{array}$ & Median \pm SD $(\mathrm{nm})$ & $1.00^{*}$ & $72.3 \pm 52.3$ & & \\
\hline $0.3 \mathrm{E}+04$ & $58.1 \pm 34.8$ & $0.50^{*}$ & $76.7 \pm 59.0$ & & \\
\hline $0.6 \mathrm{E}+04^{*}$ & $74.8 \pm 57.9$ & $0.02^{*}$ & $70.2 \pm 47.4$ & & \\
\hline $1.3 \mathrm{E}+04^{*}$ & $72.3 \pm 52.3$ & & & & \\
\hline $6.3 \mathrm{E}+04^{*}$ & $70.9 \pm 47.2$ & & & & \\
\hline
\end{tabular}

As shown in Table T1, both median and dispersion (SD) of flow-derived size distributions varied very little (especially given there can be some variability in the biological samples themselves) under optimal staining conditions (marked with a start), giving an average median and SD of $70.8 \pm 3.3 \mathrm{~nm}$ and $49.2 \pm$ $4.8 \mathrm{~nm}$, respectively (both average median and SD calculated from 16 independent experiments). 

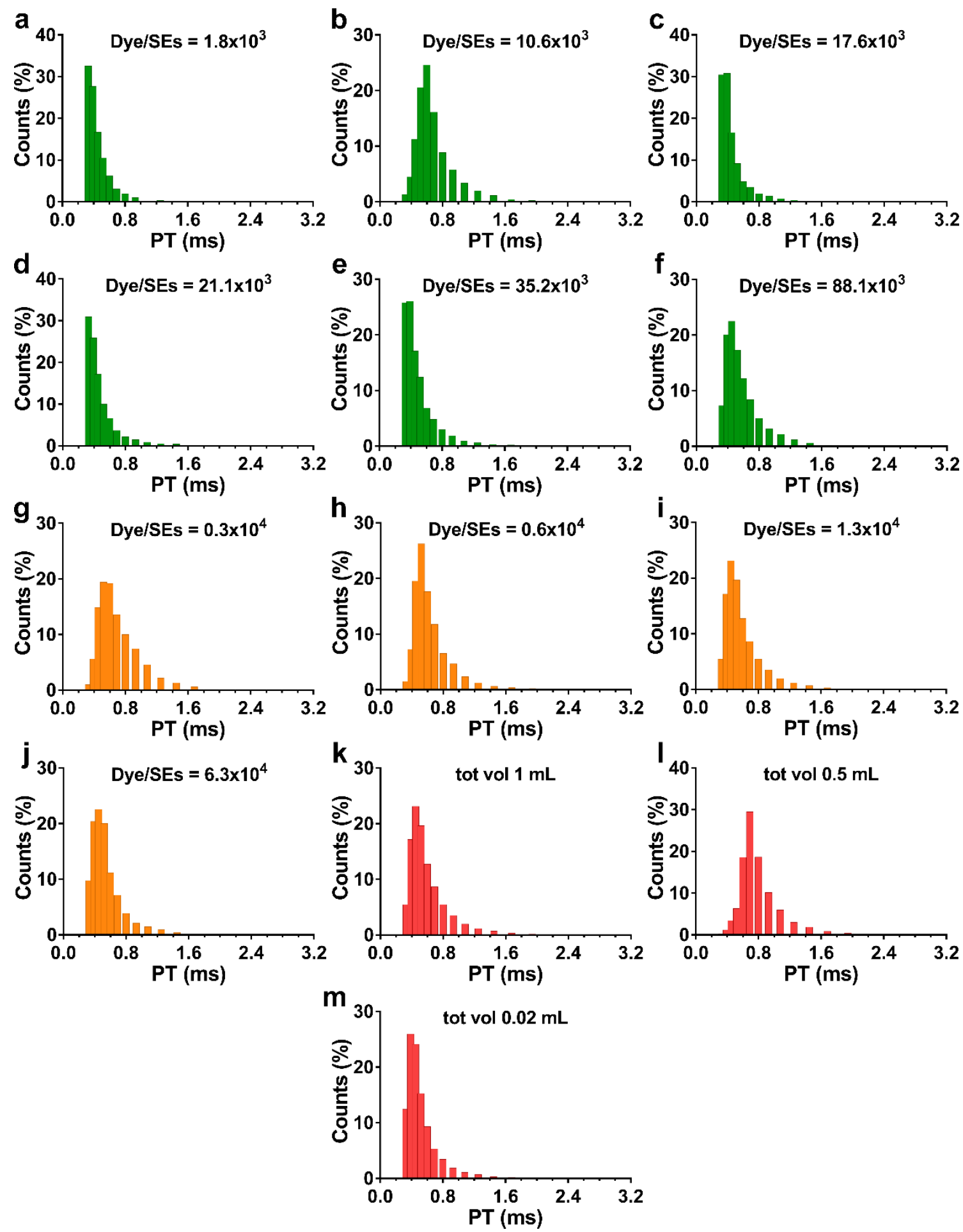

Figure S14. Distribution of SE passing times (PT). a-f, PT distributions measured during screening of Di-8-ANEPPS concentration. The distributions are centered at $0.39 \mathrm{~ms}(\mathrm{a}), 0.64 \mathrm{~ms}(\mathrm{~b}), 0.41 \mathrm{~ms}$ (c), $0.40 \mathrm{~ms}$ (d), $0.42 \mathrm{~ms}$ (e), and $0.52 \mathrm{~ms}$ (f). g-j, PT distributions measured during screening of SE concentration. The distributions are centered at $0.64 \mathrm{~ms}(\mathrm{~g}), 0.58 \mathrm{~ms}(\mathrm{~h}), 0.53 \mathrm{~ms}(\mathrm{i})$, and $0.51 \mathrm{~ms}(\mathrm{j})$. $\mathbf{k}-\mathbf{m}$, PT distributions measured during screening of the reaction volume used in SE staining. The distributions are centered at $0.53 \mathrm{~ms}(\mathrm{k}), 0.76 \mathrm{~ms}(\mathrm{l})$, and $0.47 \mathrm{~ms}(\mathrm{~m})$. 

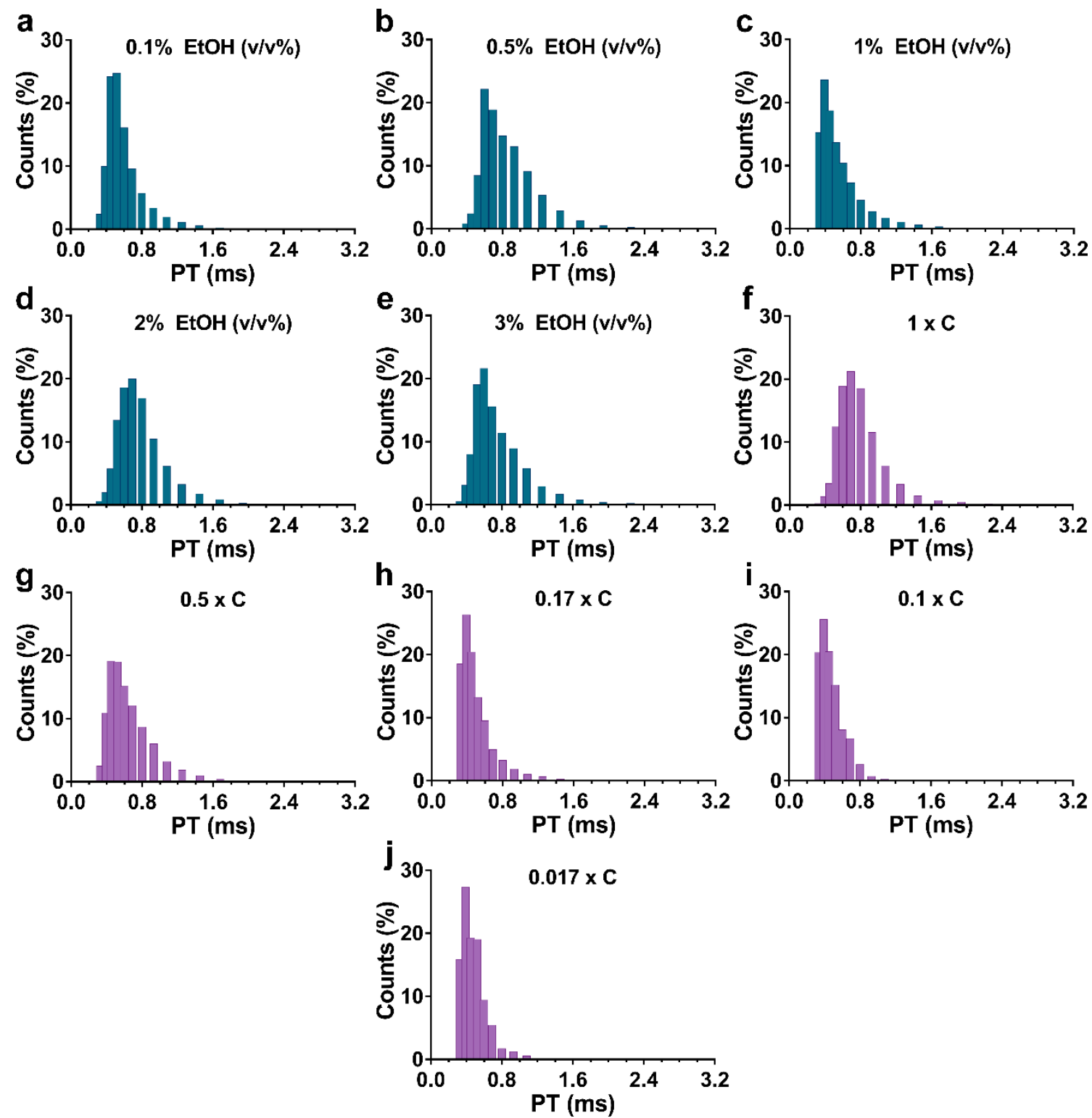

Figure S15. Distribution of SE passing times (PT). a-e, PT distributions measured during screening of EtOH concentration (v/v \%). The distributions are centered at $0.56 \mathrm{~ms}(\mathrm{a}), 0.79 \mathrm{~ms}(\mathrm{~b})$, $0.48 \mathrm{~ms}$ (c), $0.75 \mathrm{~ms}$ (d), and $0.68 \mathrm{~ms}$ (e). f-j, PT distributions measured during dilution of both Di-8ANEPPS and SEs while keeping their ratio constant. C represents the optimal concentration of SEs, $1.7 \times 10^{9}$ particles $/ \mathrm{mL}$. The distributions are centered at $0.76 \mathrm{~ms}$ (f), $0.59 \mathrm{~ms}$ (g), $0.46 \mathrm{~ms}(\mathrm{~h}), 0.44 \mathrm{~ms}$ (i), and $0.46 \mathrm{~ms}(\mathrm{j})$. 

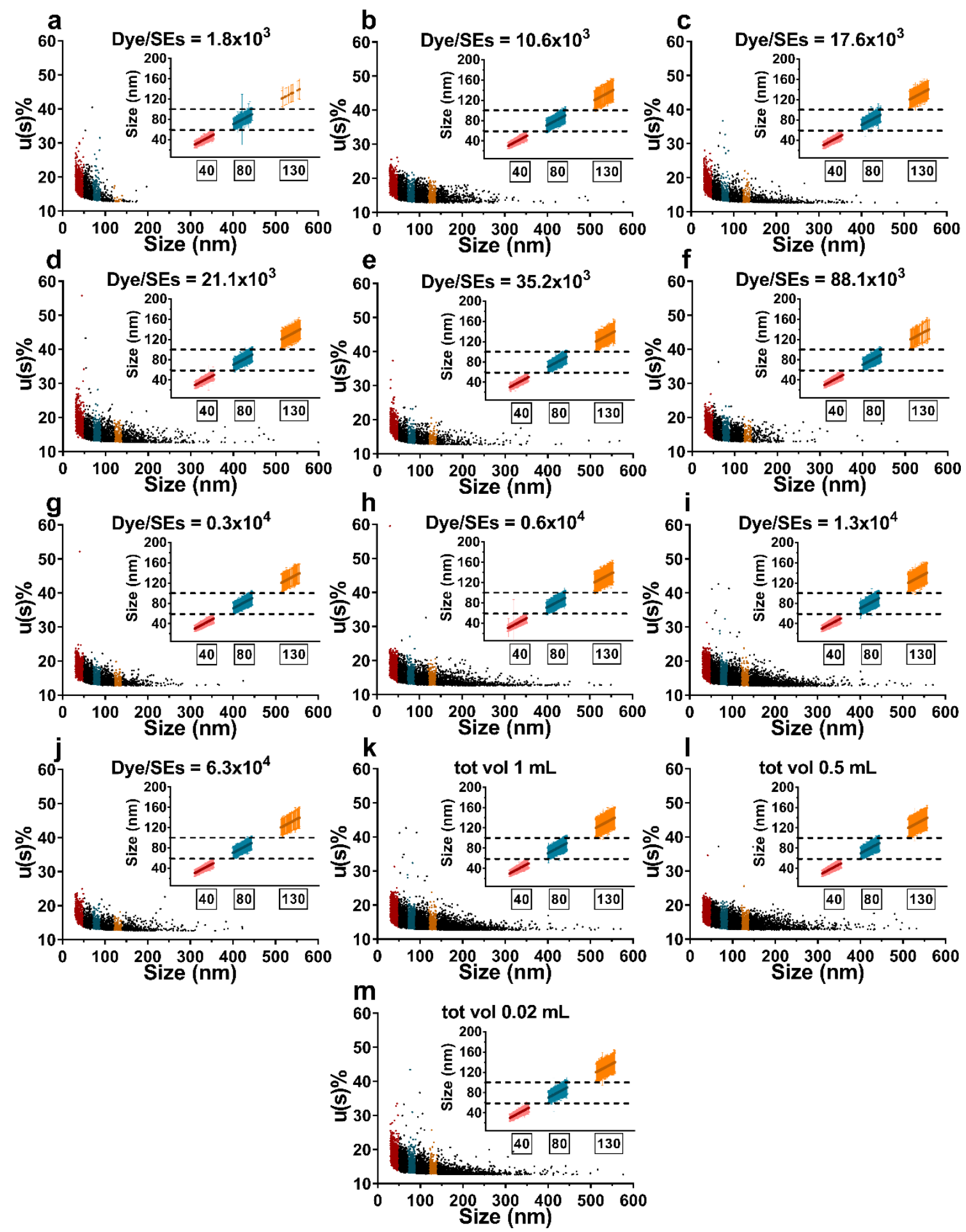

Figure S16. Scatter plots of the relative uncertainty on SE size $u(s) \%$ from different experimental conditions. The colored dots indicate three distinct subpopulations of vesicles centered at $40 \mathrm{~nm}$ (red), $80 \mathrm{~nm}$ (blue) and $130 \mathrm{~nm}$ (orange). Each subpopulation span $\pm 10 \mathrm{~nm}$ around the corresponding center. The insert shows the three subpopulations along with the absolute uncertainty (error bars in light colors) associated with each single vesicle. a-f, Scatter plots from screening Di-8-ANEPPS concentration during SE staining. g-j, Scatter plots from screening SE concentration while keeping the dye concentration constant. k-m, Scatter plots from screening total reaction volume during SE staining with Di-8-ANEPPS. 

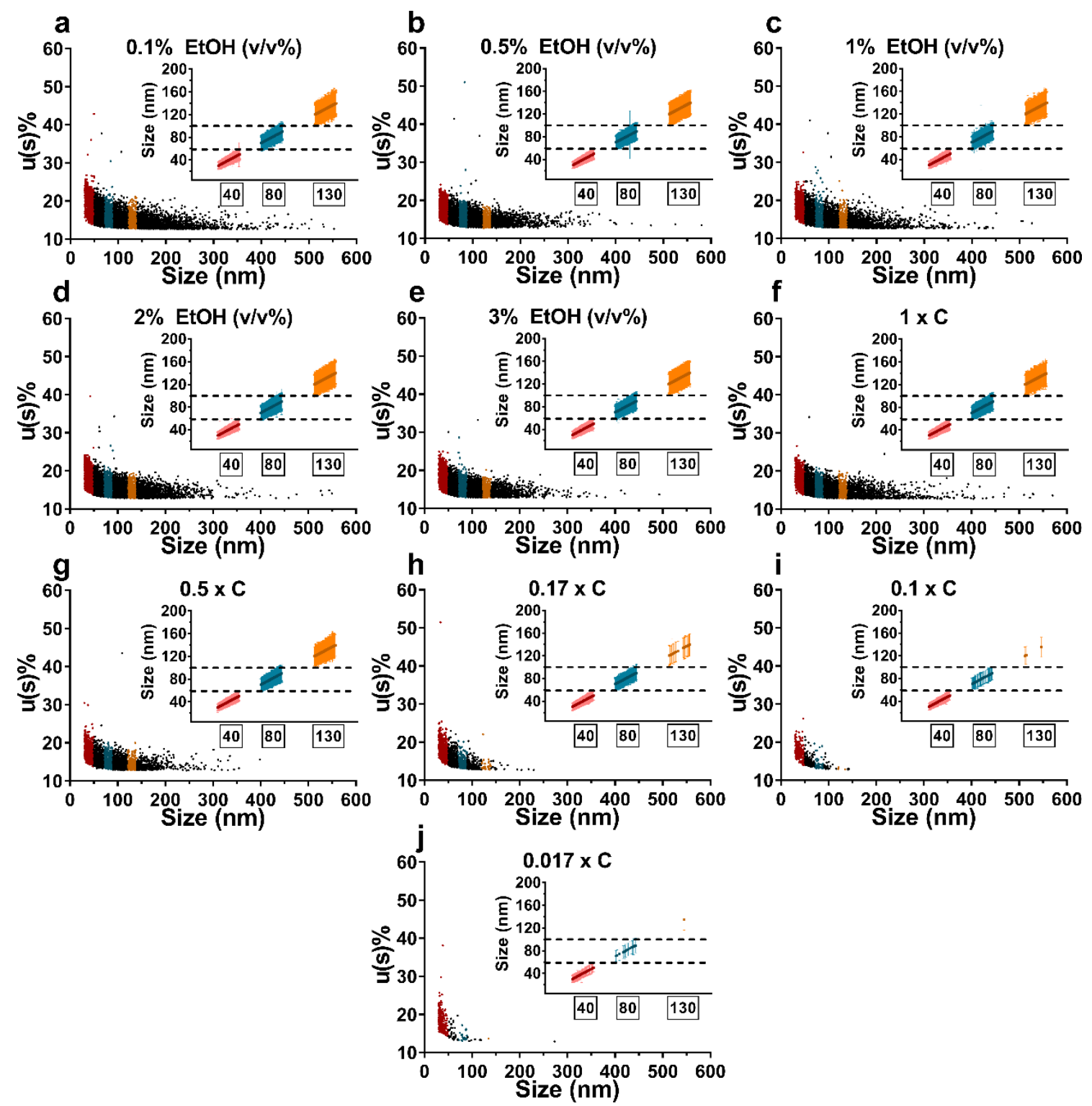

Figure S17. Scatter plots of the relative uncertainty on SE size u(s)\% from different experimental conditions. The colored dots indicate three distinct subpopulations of vesicles centered at $40 \mathrm{~nm}$ (red), $80 \mathrm{~nm}$ (blue) and $130 \mathrm{~nm}$ (orange). Each subpopulation span $\pm 10 \mathrm{~nm}$ around the corresponding center. The insert shows the three subpopulations along with the absolute uncertainty (error bars in light colors) associated with each single vesicle. a-e, Scatter plots from screening EtOH concentration (v/v \%). f-j, Scatter plots from screening of the dilution of both Di-8ANEPPS and SEs while keeping their ratio constant. C represents the optimal concentration of SEs, $1.7 \times 10^{9}$ particles $/ \mathrm{mL}$. For estimation of SE sizes, while varying the experimental conditions, we applied a cutoff at $35 \mathrm{~nm}$ (based on the analysis of S-lipo); therefore, the number of vesicles (i.e. dots) decreased considerably with the increase in dilution since the calculated size distribution shifted toward smaller sizes (see main text). 

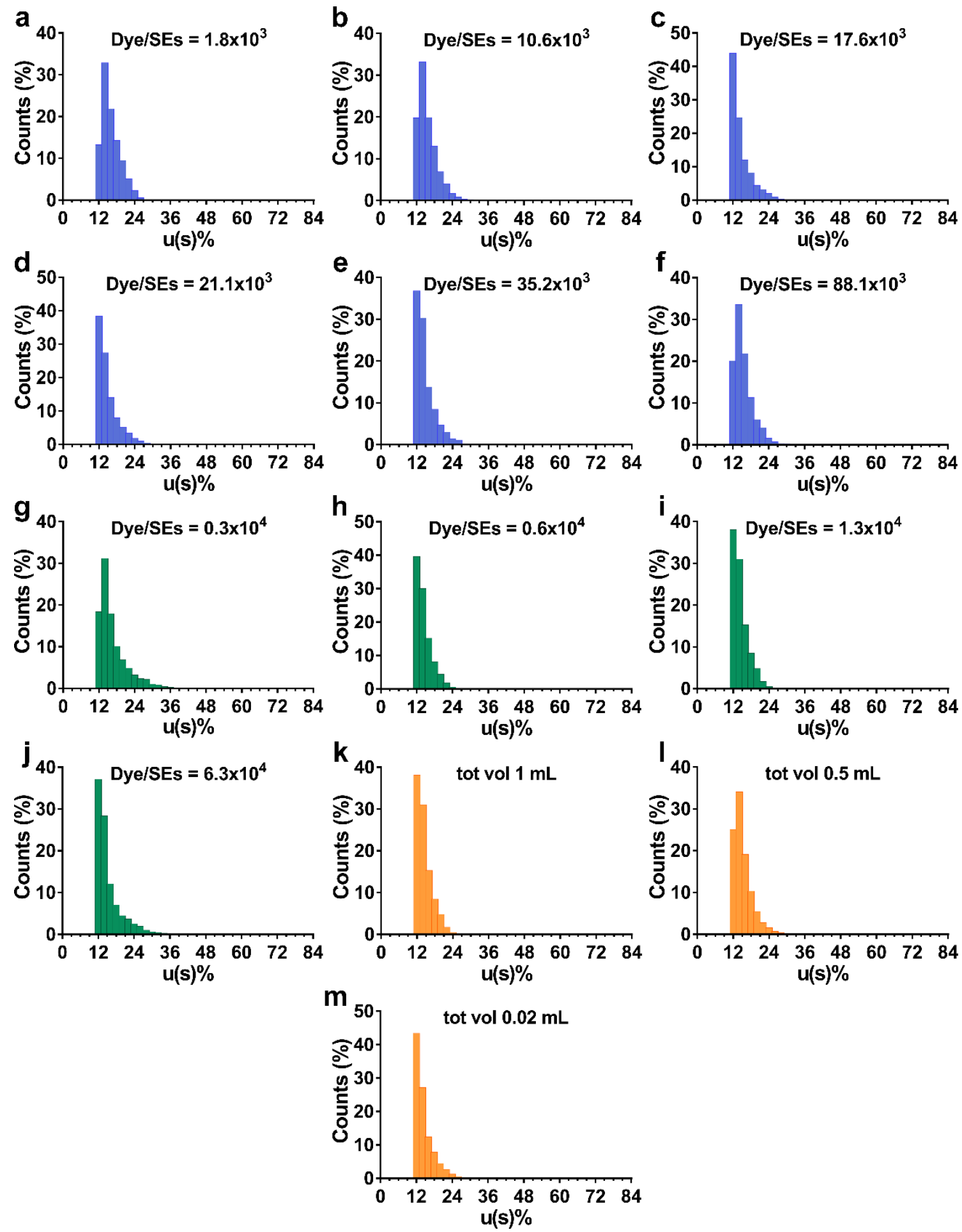

Figure S18. Distribution of relative percentage error for SEs. a-f, Distribution of relative Err\% from screening Di-8-ANEPPS concentration. g-j, Distribution of relative Err\% from screening SE concentration while keeping the amount of Di-8-ANEPPS constant. k-m, Distribution of relative Err\% from screening total reaction volume during SE staining with Di-8-ANEPPS. 

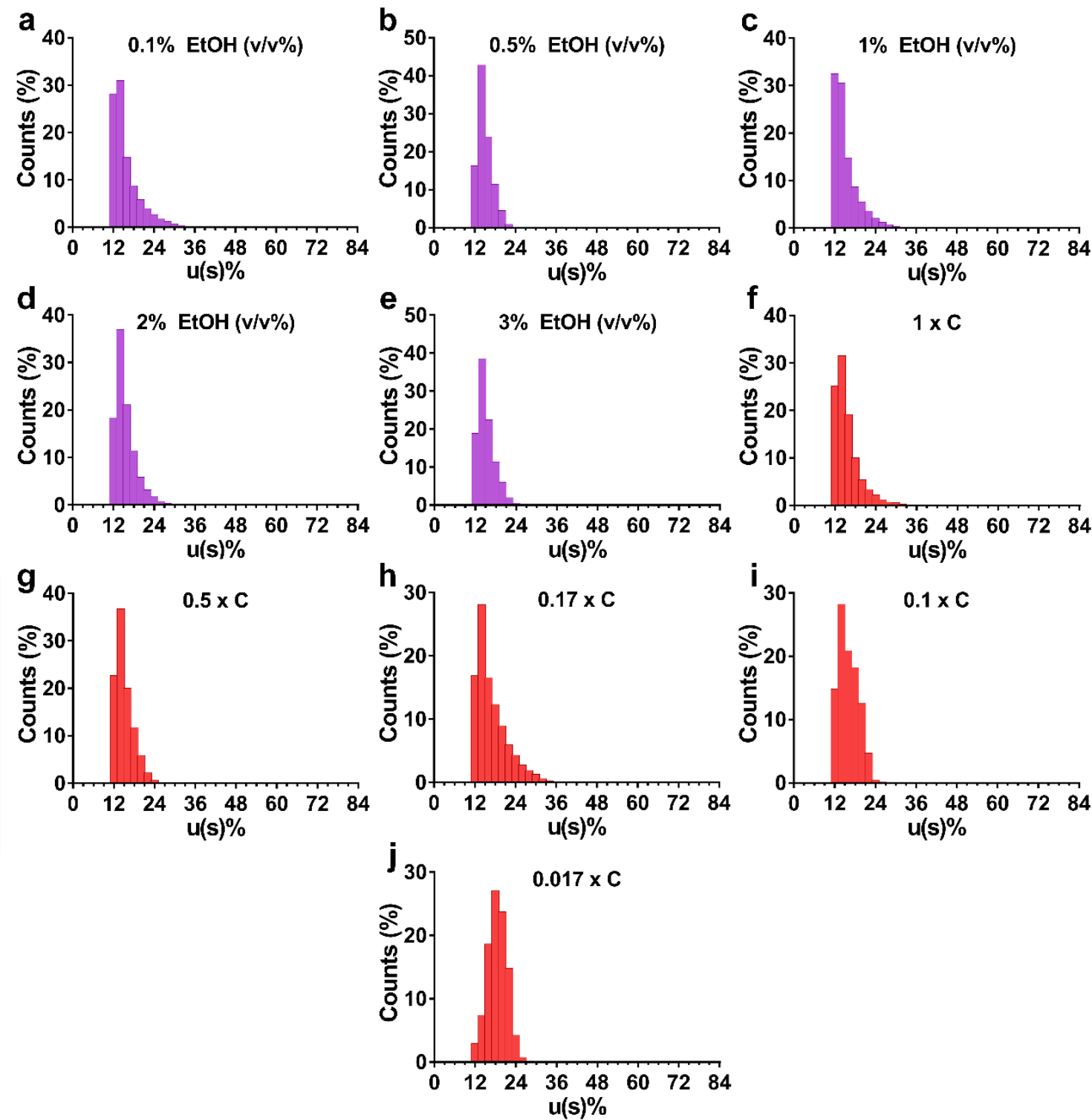

Figure S19. Distribution of relative percentage error for SEs. a-e, Distribution of relative Err\% from screening EtOH concentration (v/v \%). f-j, Distribution of relative Err\% from screening the dilution of both Di-8-ANEPPS and SEs while keeping their ratio constant. $\mathrm{C}$ represents the optimal concentration of SEs, $1.7 \times 10^{9}$ particles $/ \mathrm{mL}$. 


\section{References}

${ }^{1}$ Clarke, R. J.; Kane, D. J. Biochimica et Biophysica Acta (BBA) - Biomembranes 1997, 1323, 223-239.

2 Reeve, J. E.; et al. Biophysical Journal 2012, 103, 907-917.

${ }^{3}$ Collot, M.; et al. Cell Chemical Biology 2019, 26, 600-614.e7.

${ }^{4}$ Rusu, L.; Lumma, D.; Rädler, J. O. Macromol. Biosci. 2010, 10, 1465-1472.

5 Taylor, J. R. (1997) An Introduction to Error Analysis: The Study of Uncertainties in Physical Measurements, Edition 2, reprint, revised, Publisher University Science Books.

${ }^{6}$ Ouattara, L. A.; Anderson, S. M.; Doncel, G. F. Andrologia 2018, 50, e13220. 